\title{
A dusty filament and turbulent CO spirals in HD 135344B - SAO 206462
}

\author{
Simon Casassus, ${ }^{1 \star}$ Valentin Christiaens,${ }^{2}$ Miguel Cárcamo, ${ }^{3}$ Sebastián Perez, ${ }^{4,5}$ Philipp Weber, ${ }^{1,4}$ \\ Barbara Ercolano, ${ }^{6}$ Nienke van der Marel, ${ }^{7,8}$ Christophe Pinte, ${ }^{2}$ Ruobing Dong, ${ }^{7}$ Clément Baruteau, ${ }^{9}$ \\ Lucas Cieza, ${ }^{10}$ Ewine van Dishoeck, ${ }^{11,12}$ Daniel Price, ${ }^{2}$ Olivier Absil, ${ }^{13}$ Carla Arce-Tord, ${ }^{1}$ \\ Virginie Faramaz, ${ }^{14}$ Christian Flores, ${ }^{15}$ Maddalena Reggiani ${ }^{16}$ \\ ${ }^{1}$ Departamento de Astronomía, Universidad de Chile, Casilla 36-D, Santiago, Chile \\ ${ }^{2}$ School of Physics and Astronomy, Monash University, Clayton Vic 3800, Australia \\ ${ }^{3}$ Jodrell Bank Centre for Astrophysics, Department of Physics and Astronomy, University of Manchester, Alan Turing Building, \\ Oxford Road, Manchester, M13 9PL, UK \\ ${ }^{4}$ Departamento de Física, Universidad de Santiago de Chile. Avenida Ecuador 3493, Estación Central, Santiago, Chile \\ ${ }^{5}$ Center for Interdisciplinary Research in Astrophysics and Space Exploration (CIRAS), Universidad de Santiago de Chile \\ ${ }^{6}$ Universitäts-Sternwarte, Ludwig-Maximilians-Universität München, Scheinerstr. 1, 81679 München, Germany \\ ${ }^{7}$ Department of Physics \& Astronomy, University of Victoria, Victoria, BC, V8P 1A1, Canada \\ ${ }^{8}$ Banting Research fellow \\ ${ }^{9}$ IRAP, Université de Toulouse, CNRS, UPS, Toulouse, France \\ ${ }^{10}$ Núcleo de Astronomía, Facultad de Ingeniería y Ciencias, Universidad Diego Portales, Av Ejército 441, Santiago, Chile \\ ${ }^{11}$ Leiden Observatory, Faculty of Science, Leiden University, PO Box 9513, 2300 RA Leiden, The Netherlands \\ ${ }^{12}$ Max-Plank-Institut fur Extraterrestrische Physik, Giessenbachstrasse 1, D-85748 Garching,Germany ${ }^{13}$ STAR Institute, Université de Liège, $19 \mathrm{c}$ Allée du Six Août, 4000 L \\ ${ }^{14}$ Jet Propulsion Laboratory, California Institute of Technology, 4800 Oak Grove drive, Pasadena CA 91109, USA \\ ${ }^{15}$ Institute for Astronomy, University of Hawaii at Manoa, 640 N. Aohoku Place, Hilo, HI 96720, USA \\ ${ }^{16}$ Institute of Astronomy, KU Leuven, Celestijnenlaan 200D, B-3001 Leuven, Belgium.
}

Accepted XXX. Received YYY; in original form ZZZ

\begin{abstract}
Planet-disc interactions build up local pressure maxima that may halt the radial drift of protoplanetary dust, and pile it up in rings and crescents. ALMA observations of the HD 135344B disc revealed two rings in the thermal continuum stemming from $\sim \mathrm{mm}$-sized dust. At higher frequencies the inner ring is brighter relative to the outer ring, which is also shaped as a crescent rather than a full ring. In near-IR scattered light images, the disc is modulated by a 2-armed grand-design spiral originating inside the ALMA inner ring. Such structures may be induced by a massive companion evacuating the central cavity, and by a giant planet in the gap separating both rings, that channels the accretion of small dust and gas through its filamentary wakes while stopping the larger dust from crossing the gap. Here we present ALMA observations in the $J=(2-1)$ CO isotopologue lines and in the adjacent continuum, with up to $12 \mathrm{~km}$ baselines. Angular resolutions of $\sim 0$.' 03 reveal the tentative detection of a filament connecting both rings, and which coincides with a local discontinuity in the pitch angle of the IR spiral, proposed previously as the location of the protoplanet driving this spiral. Line diagnostics suggest that turbulence, or superposed velocity components, is particularly strong in the spirals. The ${ }^{12} \mathrm{CO}(2-1) 3-\mathrm{D}$ rotation curve points at stellocentric accretion at radii within the inner dust ring, with a radial velocity of up to $\sim 6 \% \pm 0.5 \%$ Keplerian, which corresponds to an excessively large accretion rate of $\sim 2 \times 10^{-6} M_{\odot} \mathrm{yr}^{-1}$ if all of the $\mathrm{CO}$ layer follows the ${ }^{12} \mathrm{CO}(2-1)$ kinematics. This suggests that only the surface layers of the disc are undergoing accretion, and that the line broadening is due to superposed laminar flows.
\end{abstract}

Key words: protoplanetary discs — accretion, accretion discs — planet-disc interactions

\section{INTRODUCTION}

The radial drift of protoplanetary dust halts at local pressure maxima (Weidenschilling 1977), where the azimuthal gaseous flow exerts no net drag. The pile-up of dust with dimensionless stopping time (Stokes number) $S_{t} \lesssim 1$ in radial pressure bumps, whichever their origin, explains the Atacama Large Millimeter/submillimeter Array (ALMA) observations of dusty ringed systems, such as reported in

\footnotetext{
^ E-mail: simon@das.uchile.cl
}

HLTau (ALMA Partnership et al. 2015; Carrasco-González et al. 2019), in HD 169142 (e.g. Pérez et al. 2019; Sierra et al. 2019), or in the DSHARP surveys (Andrews et al. 2018; Dullemond et al. 2018).

The same radial pressure discontinuities leading to radial trapping can also trigger the Rossby-wave instability, and develop a large scale anti-cyclonic vortex (Lovelace et al. 1999; Li et al. 2001) resulting in strong radial and azimuthal concentration for $S_{t} \lesssim 1$ dust grains (Birnstiel et al. 2013; Lyra \& Lin 2013; Zhu \& Stone 2014; Mittal \& Chiang 2015; Baruteau \& Zhu 2016). The radial pressure discontinuity itself could result from the formation of a planetary gap (Zhu 
\& Stone 2014; Koller et al. 2003; de Val-Borro et al. 2007; Zhu \& Baruteau 2016), among other possibilities (e.g. Varnière \& Tagger 2006; Regály et al. 2012).

Azimuthal dust traps have been identified observationally with ALMA in the form of large-scale crescents of continuum submm emission, with extreme azimuthal contrast ratios of $\sim 30$ in HD 142527 (Casassus et al. 2013, 2015; Muto et al. 2015; Boehler et al. 2017) and 100 in IRS 48 (van der Marel et al. 2013, 2015b; Ohashi et al. 2020). Such extreme lopsidedness, in combination with an otherwise full gas disc as revealed by CO observations, has been interpreted as likely due to dust trapping in a vortex (Birnstiel et al. 2013; Lyra \& Lin 2013; Baruteau \& Zhu 2016; Sierra et al. 2017). Finer ALMA angular resolutions have revealed that crescents with varying contrast ratios are fairly frequent in the outer rings of ringed systems, such as in LkH $\alpha 330$ (Isella et al. 2013), SR 21, HD135344B (Pérez et al. 2014; van der Marel et al. 2015a, 2016b; Cazzoletti et al. 2018), DoAr 44 (van der Marel et al. 2016a), and HD 34282 (van der Plas et al. 2017). The occurrence of such asymmetries is associated with Stokes numbers $S_{t} \lesssim 1$, in agreement with the dust trapping scenario (e.g. van der Marel et al. 2021). In the brighter clump of MWC 758 at VLA frequencies, the multi-frequency continuum observations can be reproduced with the Lyra-Lin trapping prescriptions, which yields estimates of the local physical conditions and constraints on the dust population (Marino et al. 2015; Casassus et al. 2019).

Similar processes as in protoplanetary discs are thought to have occurred in the protosolar disc. The statistics of meteoritic inclusions provide important information on the physical processes that shaped the Solar System. The same gapped system predicted for an accreting proto-Jupiter also accounts for the filtering of the larger dust grains out of the inner disc, and explains the size distributions of meteoritic inclusions (Haugbølle et al. 2019). Multi-fluid simulations including dust and gas show that the smaller dust population sieves across the proto-jovian gap through the planetary wakes, i.e. as in the bottleneck of an hourglass (e.g. Weber et al. 2018) which prevents the larger dust from crossing the gap, with the maximum grain size permitted to cross depending on disc characteristics (Weber+2018).'

It is interesting to search for similar features in exo-protoplanetary systems as required to explain the meteoritic inclusions: smaller dust grains in the inner regions of protoplanetary discs hosting deep gaps. Such inner dust discs are frequently detected, and appear to be depleted in mm-dust mass (e.g. Francis \& van der Marel 2020).

The disc around HD 135344B, also called SAO 206462 (with a spectral type F8V, and at a distance of $135.7 \pm 1.4$ pc Gaia Collaboration et al. 2018), is particularly interesting because of several planet-formation signposts, as its sub-mm continuum emission is essentially composed of two rings, at 0." 4 (52 au) and 0.'6 (80 au) separated by a deep and broad gap, and crossed by a grand-design 2-armed spiral prominent in scattered-light images (Stolker et al. 2016). The two arms of this spiral pattern have been proposed to be driven by two planets orbiting at $\sim 00^{\prime \prime} 4$ and $\sim 0$.' 9 (so $\sim 54$ au and $\sim 121.5 \mathrm{au}$, Muto et al. 2012), or from a massive planet inside the cavity at $\sim 0$.'23 along with the outer disc vortex (van der Marel et al. 2016a,b), or the whole pattern could result from gravitational instability (GI, Dong et al. 2015, 2018b). Multi-epoch monitoring of the scattered-light spirals has allowed the measurement of the pattern motion, which points at a companion at $86_{-13}^{+18}$ au if the arms are comoving, or else to two companions at $49_{-5}^{+6}$ au and $120 \pm 30$ au (Xie et al. 2021). The multi-wavelength imaging reported by Cazzoletti et al. (2018) supports an interpretation in terms of the filtering of the larger dust grains out of the inner regions, since the inner ring is increasingly brighter at higher frequencies compared to the outer ring. This brightness effect could also point to a difference in dust evolution within the two rings, i.e. to different fragmentation rates.

Here we report on new ALMA observations of HD 135344B (Section 2) that reveal the tentative detection of the dusty protoplanetary wakes predicted by theory. A narrow trickle of dust continuum emission seems to connect the lopsided outer ring with the inner ring (Sec. 3), close to the planet at $\sim 0{ }^{\prime \prime} 4$ proposed by Muto et al. (2012). A simple analysis of the line emission reveals that the 2-armed spiral corresponds to enhanced velocity dispersions (Sec. 3). We interpret the available data in terms of disc-surface accretion towards the star (Sec. 4).

\section{OBSERVATIONS}

HD 135344B was observed with ALMA during Cycle 6, as part of program 2018.1.01066.S. The data presented in this article correspond to a partial delivery of the whole program, which is scheduled for execution in Cycle 7 with a nominal $13 \mathrm{~h}$ of telescope time. In this partial dataset, HD 135344B was observed from 13Jul-2019/02:43:39 to 13-Jul-2019/03:55:34 (UTC), for a total of $87 \mathrm{~min}$ on-source. The phase centre of the array pointed at J2000 $15 \mathrm{~h} 15 \mathrm{~m} 48.4142 \mathrm{~s}-37 \mathrm{~d} 09 \mathrm{~m} 16.4776 \mathrm{~s}$, which is offset by 5.8 mas from the ICRS position of the star at the epoch of observations, J2000 $15 \mathrm{~h} 15 \mathrm{~m} 48.4147 \mathrm{~s}-37 \mathrm{~d} 09 \mathrm{~m} 16.4785 \mathrm{~s}$. The array counted 43 active antennas, with baselines ranging from $111.2 \mathrm{~m}$ to $12.6 \mathrm{~km}$. The column of precipitable water vapour (PWV) ranged from $0.9 \mathrm{~mm}$ at the beginning of the integration, to $0.7 \mathrm{~mm}$ at the end. The correlator was setup to provide 4 spectral windows (spws): spw 0 , sampling the continuum around $218 \mathrm{GHz}$ with 128 channels over a total bandwidth of $2 \mathrm{GHz}$; spw 1, for ${ }^{12} \mathrm{CO}(2-1)$ at a rest frequency of $230.538 \mathrm{GHz}$, sampled with $158.74 \mathrm{~m} \mathrm{~s}^{-1}$ channels; spw 2, sampling the continuum around $232 \mathrm{GHz}$ with 960 channels over a total bandwidth of $1.875 \mathrm{GHz}$; spw 3 , for $\mathrm{C}^{18} \mathrm{O}(2-1)$ at a rest frequency of $219.560 \mathrm{GHz}$, sampled with $166.68 \mathrm{~m} \mathrm{~s}^{-1}$ channels; spw 4 , for ${ }^{13} \mathrm{CO}(2-1)$ at a rest frequency of $220.399 \mathrm{GHz}$, sampled with $166.04 \mathrm{~m} \mathrm{~s}^{-1}$ channels. The data were calibrated by staff from the North America ALMA Regional Center.

Spectral windows spw 0 and spw 2 were devoid of conspicuous line emission and were combined to image the continuum. A single self-calibration loop, setting up CASA task gaincal to average whole scans (option 'solint' set to 'inf'), provided an improvement in tclean images using Briggs weights with robustness parameter $r=0.0$, with a dynamic range (i.e. signal-to-noise ratio) increasing from $\sim 14.8$ to $\sim 17.3$. The quantitative improvement was small but the self-calibration loop got rid of noisy patches, so we adopted the selfcalibrated dataset. We then applied the UVMEM package (Casassus et al. 2006; Cárcamo et al. 2018) on the self-calibrated continuum data to produce a non-parametric model image. In brief, UVMEM produces a model-image $I_{j}^{m}$ and model visibilities $V_{k}^{m}$ that fit the visibility data $V_{k}^{\circ}$ in a least-square sense, with the possible inclusion of a regularising term, by minimising a figure of merit $L$ :

$L=\sum_{k=1}^{N} \omega_{k}\left|V_{k}^{\circ}-V_{k}^{m}\right|^{2}+\lambda S$,

where $\omega_{k}$ correspond to the visibility weights. In this case image positivity provided sufficient regularisation, so we otherwise restricted the optimisation to the least-squares term only (see for example Casassus et al. 2018, 2019, for a detailed applications of UVMEM to ALMA data in protoplanetary discs). 
As summarised in Fig. 1, the continuum data are consistent with the general structure of the disc previously reported by Cazzoletti et al. (2018). The disc is composed of two main features: an outer ring is shaped into a large crescent, as expected for a vortex (e.g. Baruteau \& Zhu 2016), and an inner ring whose brightness relative to the outer ring increases with frequency. Here we also notice a faint plateau or pedestal abut onto the inner ring, and enclosing an otherwise very deep cavity. The central source, probably related to the star, shows signal at $5 \sigma$, with intriguing structure the details of which should be ascertained in second epoch imaging.

When integrated over the whole field plotted in Fig. 1, the continuum flux density at $225 \mathrm{GHz}$ from HD $135344 \mathrm{~B}$ is $117.4 \pm 0.1 \mathrm{mJy}$ in the Briggs 2.0 image, with a noise of $\sim 18 \mu \mathrm{Jy}_{\text {beam }}{ }^{-1}$, and $105.1 \pm$ $0.3 \mathrm{mJy}$ in the Briggs 0.0 image, with a noise of $\sim 25 \mu \mathrm{Jy} \mathrm{beam}^{-1}$. The thermal uncertainty is very small, but both values are affected by a systematic uncertainty of $\sim 10 \%$ root-mean-square (rms). The nominal maximum recoverable angular scale (MRS) for this array configuration is $\sim 0$.' 62 (as given in the ALMA proposer's guide for Cycle 6 ), and since the continuum signal extends over $\sim 11^{\prime \prime} 2$, there may be a measure of flux loss, i.e. missing low spatial frequencies in the reconstructed image due to missing short-spacings in the $u v$-coverage. The slightly lower flux in Briggs 0.0 compared to Briggs 2.0 may perhaps reflect this flux loss effect. But the total missing flux must be fairly small, as there are no obvious image synthesis artefacts that modulate the signal.

The line datacubes were extracted following a standard procedure, and after propagating the self-calibration gain tables to the full dataset. Continuum subtraction was performed using CASA task uvcontsub, and using a linear model that avoids the vicinity of the lines. The data were then resampled into the local standard of rest, and using spectral channels with a width of $0.2 \mathrm{~km} \mathrm{~s}^{-1}$, common to all lines. The resulting datacubes were imaged with CASA task tclean, in its multi-scale version, with automatic masking (as implemented in the 'auto-multithresh' option to tclean), and using Briggs $r=2.0$. In the case of the ${ }^{12} \mathrm{CO}(2-1)$ line, the peak signal is $\sim 16 \mathrm{mJy}$ beam $^{-1}$, while the noise (including image synthesis residuals) is $2.2 \mathrm{mJy} \mathrm{beam}^{-1}$ (for a beam 0 ' $^{\prime} 054 \times 00^{\prime \prime} 040 / 89.4 \mathrm{deg}^{1}$ ). The rarer isotopologues are much fainter than ${ }^{12} \mathrm{CO}(2-1)$, especially beyond the inner ring. We smoothed the data with a circular Gaussian taper, applied in the $u v$-plane, with a width of 60 mas. The resulting tapered beam is $\sim 0$.' $^{\prime} 12 \times 00^{\prime \prime} 10 /-88 \mathrm{deg}$ (see caption to Figs. 2 ).

The moments maps shown in Figs. 2 were extracted using singleGaussian fits to each datacube using package GMoments ${ }^{2}$. This package fits the line profile in each line of sight with either one or two Gaussians, and uses these model Gaussian line profiles to calculate the velocity moments. For a single Gaussian with $I_{v}=A \exp \left(-\frac{v-\bar{v}}{\sigma_{v}}\right)$ the line intensity is given by moment 0 , or $I=\sqrt{2 \pi} A \sigma$, while the velocity centroid matches the Gaussian centroid $\bar{v}$. We note the two-armed grand-design spiral that stands out in the ${ }^{12} \mathrm{CO}$ velocityintegrated intensity and velocity dispersion, but that is absent in the line peak intensity. These ${ }^{12} \mathrm{CO}$ data do not appear to be affected by the underlying continuum in the large crescent: continuum subtraction does not lead to any local decrement in the tapered images, so that ${ }^{12} \mathrm{CO}(2-1)$ emission is seen to extend out to radii of $\sim 1$ l. $^{\prime} 1$.

The integrated ${ }^{12} \mathrm{CO}(2-1)$ signal is more extended and smoother than the continuum, and may be affected by flux-loss. However,

\footnotetext{
1 expressed in the form (BMAJ $\times$ BMIN / BPA), where BMAJ and BMIN are the full-width major and minor axis, and BPA is the beam PA in degrees East of North.

2 https://github.com/simoncasassus/GMoments
}

the channel maps do not appear to show any spurious effects due to missing low spatial frequencies. The impact of flux-loss will be quantified with the delivery of the full dataset.

We also applied UVMEM to the ${ }^{12} \mathrm{CO}(2-1)$ data, with an entropic term for regularisation as the data are quite noisy. We minimised $L$ in Eq. 1 with

$S=\sum_{j=1}^{M} \frac{I_{j}^{m}}{M} \ln \left(\frac{I_{j}^{m}}{M}\right)$,

where $M$ is the default pixel intensity value, and is set to $10^{-3}$ times the theoretical noise of the dirty map (as inferred from the visibility weights). Here we use a control factor $\lambda=3 \times 10^{-3}$. The restored data cubes, obtained using the UVMEM model datacube and Briggs $r=2$, yield a similar dynamic range as with tclean, but are less clumpy and more sensitive to extended signal. The data from the rarer isotopologues were too noisy to allow any meaningful improvement with UVMEM.

The top and bottom ${ }^{12} \mathrm{CO}(2-1)$ layers are sufficiently separated in this disc to trace each layer with a double-Gaussian fit, as summarised in Fig. 3 for the UVMEM-restored datacube. We assume that the brighter of the two Gaussians traces the top side, that faces the observer. The velocity-integrated intensity is very similar to the single-Gaussian case, as is the Gaussian velocity dispersion. Interestingly the disc PA inferred from the velocity centroid of the brighter Gaussian, $v_{1}^{\circ}$, shifts slightly to the North-West with increasing distance from the star, as expected for the surface of a cone in which the side nearest to the observer is to the South-East. However, the velocity centroid $v_{2}^{\circ}$ corresponding to the fainter Gaussian, shifts progressively to the South-East, indicating that it is indeed tracing the bottom layer. This suggests that the extended disc and the spiral modulation seen in dispersion is intrinsic to the top (or bottom) layer, and is not the result of broadening due to a second velocity component stemming from the bottom layer.

\section{ANALYSIS}

\subsection{Continuum filament and disk orientation}

The $225 \mathrm{GHz}$ continuum image reported in Fig. 1 reveals interesting fine structure in the gap that divides the inner and outer rings. A very fine filament appears to join the two rings, at a radial separation of $\sim 0$ !' 468 (63.5 au) and PA 216.1 deg, as highlighted in Fig. 1 with yellow circles. It is best seen in the tclean image with Briggs $r=0$, where the median intensity in a region isolating the filament is $104 \mu \mathrm{Jy}_{\text {beam }^{-1}}$, and the image noise is $24 \mu \mathrm{Jy}_{\text {beam }}{ }^{-1}$. While this would appear as a $4 \sigma$ detection, there are other fine features in the same gap at a similar intensity level, although these other features are smaller and appear to sprout away from either the inner or outer ring (and may also be real). These other features suggest that the detection of this filament should be considered as a tentative result, whose confirmation requires deeper observations. We note however in Sect. 3.2 the almost coincidental location of the filament with a twist observed in the near-IR spirals reported in Muto et al. (2012) and Stolker et al. (2016), which suggests the filament may indeed be tracing a gap-crossing planetary wake.

Fig. 4 compares the ALMA continuum image of HD 135344 B with the polarised intensity image of the disc that was acquired with VLT/SPHERE on 2016-06-30. This $Q_{\phi}$ image (see definitions in Avenhaus et al. 2014; Garufi et al. 2014) of HD 135344 B is the one obtained in the best seeing conditions (average $0.5-\mu \mathrm{m}$ seeing of $0 .$. 37; Stolker et al. 2017), and is therefore favoured throughout 

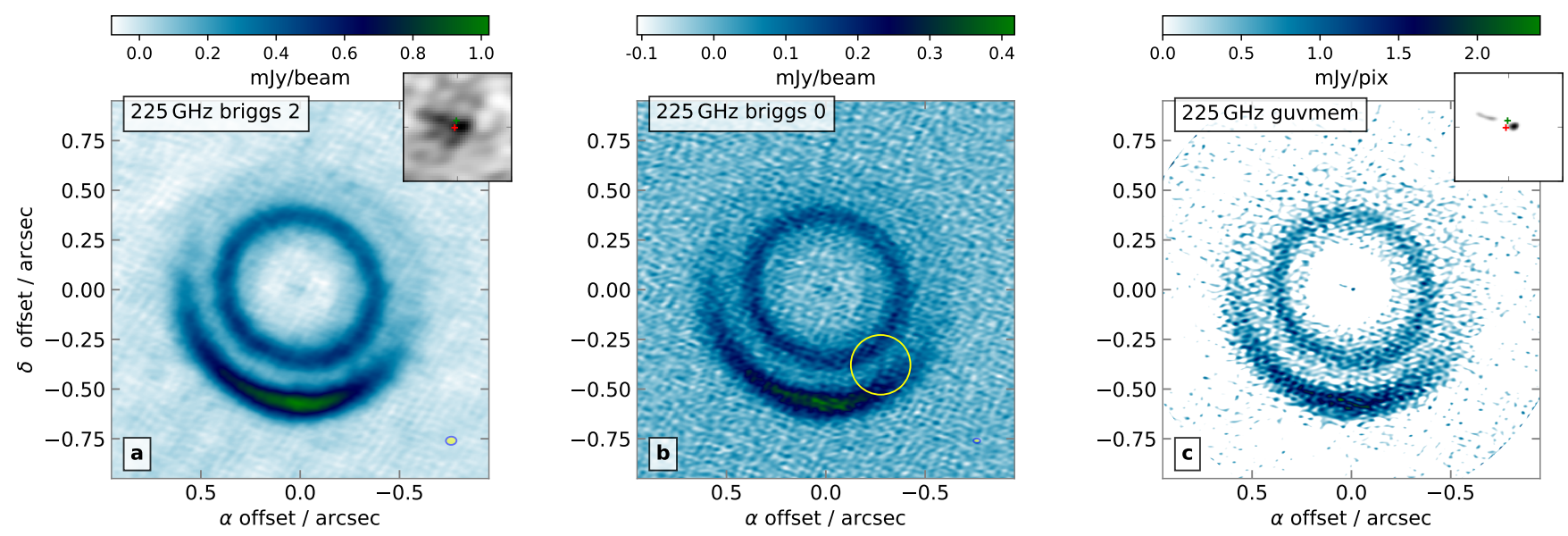

Figure 1. Continuum imaging in HD 135344B. a: CASA-tclean image with Briggs robustness parameter $r=2.0$ (so close to natural weights), resulting in a clean beam of $0 . \prime 054 \times 0$ " $041 /-87 \mathrm{deg}$, where we give the beam major axis (bmaj), minor axis (bmin) and direction (bpa) in the format bmaj $\times$ bmin/bpa. b: CASA-tclean image with Briggs robust parameter 0.0, with a clean beam $0{ }^{\prime \prime} 034 \times 0 . \prime 022 / 88$ deg c: uvmem model image, with an effective angular resolution of $\sim 1 / 3$ of the natural-weights beam, or $\sim 0 \prime^{\prime \prime} 018 \times 0 . \prime 014 /-87 \mathrm{deg}$. The yellow circles in all plots highlight the tentative detection of filamentary emission, possibly due to a planetary wake. They are centred on $[-0,276,-0.378]$. The insets zoom inside the central emission, with sides of 0 .' 2 : the origin of coordinates is marked by a red symbol, that should coincide with the position of the star at the epoch of observation for a perfect ALMA pointing accuracy, and the centroid of the inner ring is marked by a green symbol (see Sec. 3.1 for details).

the rest of this work for comparison to our ALMA data. We rereduced this SPHERE/IRDIS dataset with the IRDAP pipeline (van Holstein et al. 2020) to produce the image shown in Fig. 4 a. We see in Fig. $4 \mathrm{~b}$ that the contours that trace the $Q_{\phi}$ image come very close to the (possible) radio-mm filament. While we leave a detailed comparison between the filament and the spiral arms to Sect. 3.2, Fig. 4b shows another interesting similarity between the radio-mm and IR in this source. The faint continuum pedestal abut inside the inner ring appears to surround the bright inner ring in polarised intensity. Such faint pedestals are also seen in other systems, as, for example, in the rings of DoAr 44 and RXJ 1633.9 (called inflection points in Cieza et al. 2021) and in PDS 70 (Isella et al. 2019).

The continuum images can be used to infer disc orientation. We minimised the scatter in the radial profile, extracted by averaging in azimuth over the radial range [0,'25, 0,'45] (so enclosing only the inner ring). The procedure involves 4 free-parameters: the disc position angle (PA), inclination $i$, and the origin for the polar expansions, which is offset relative to the origin of coordinates in the images by $\Delta \alpha$ in right-ascension and $\Delta \delta$ in declination. We used the MPoLARMAPS package, which is described in appendix. The posterior distributions were sampled with the EMCEE package (Foreman-Mackey et al. 2013), using flat priors. The disc orientation resulting from the tclean image with $r=0$ Briggs weights is $\mathrm{PA}=241.6_{-6.0}^{6.9} \mathrm{deg}$, $i=23.80_{-2.6}^{2.52} \mathrm{deg}, \Delta \alpha=2_{-4}^{3}$ mas, $\Delta \delta=12_{-4}^{4}$ mas. The origin of the polar expansion is plotted with a green marker in Fig. 1. We note that the disc PA is consistent with that used by Cazzoletti et al. (2018), considering that here we point PA at the position of the ascending node, but the disc inclination inferred in this work is significantly higher. A lower inclination is also favoured by the line data (when comparing against independent measurements of the stellar mass, see Sec.3.4). The high inclination resulting from this analysis of the continuum may reflect that the inner ring is intrinsically eccentric. The face-on views in Fig. 5 show that the present inclination results in a circular inner ring, albeit offset from the central emission. The orientation that corresponds to the most axially symmetric ring results in a scatter for the azimuthal profile of the ring radius of $\sigma\left(r_{\text {cav }}\right)=5$ mas about a median of $0 . ' 38$. However, using a lower inclination of $i=16 \mathrm{deg}$ results in a pronounced eccentricity in the deprojected (face-on) views, which is more obvious in the polar expansions, with an azimuthal scatter $\sigma\left(r_{\text {cav }}\right)=8$ mas, about a median of 0. " 37 .

As discussed below, an inclination close to $i=16 \mathrm{deg}$ is required to bring the dynamical mass of the star in agreement with photospheric measurements. The difference with the inferred inclination of $23.8 \mathrm{deg}$ based on the inner dust ring suggest that the inclination difference, of $\sim 7.8 \pm 2.5$, is due to intrinsic ring eccentricity, which corresponds to $e=0.14 \pm 0.04$. This value is comparable to the measurement of $e \sim 0.1$ in MWC 758 by Dong et al. (2018a).

\subsection{Spiral arms}

Both the Gaussian and tapered versions of the ${ }^{12} \mathrm{CO}(2-1)$ moment 0 and 2 maps reveal a double-armed spiral pattern (Figs. 2 and 3), reminiscent of the morphology of the near-IR spirals reported in Muto et al. (2012); Stolker et al. (2016). In order to facilitate the interpretation of these spirals, we identified their trace in both the ${ }^{12} \mathrm{CO}(2-1)$ moment 0 map and the IR image, fitted their traces to different spiral equations, and compared the best-fit models with the $\mathrm{CO}$ moment 0 map, the IR image and the sub-mm continuum image (Fig. 6).

We first identified local radial maxima in 1-deg wide slices in the Gaussian moment 0 map (where the spirals are the most conspicuous), after subtraction of the median radial intensity profile. These median intensities are integrated azimuthally over concentric 1-FWHM wide ellipses for all pixels, considering the mid-plane orientation of the disc inferred from the CO rotation curves (Sect. 3.3; $i \sim 17.6 \mathrm{deg}$ and PA $241.9 \mathrm{deg}$ ). In each azimuthal slice, the vicinity of each radial maximum identified is then fitted to a 1D Gaussian profile using SCIPY's curve_fit routine. For each position angle, the radial separation and associated uncertainty on the spiral trace are set as the centroid of the Gaussian, and the maximum between the uncertainty on the centroid and half the beam size of our ob- 

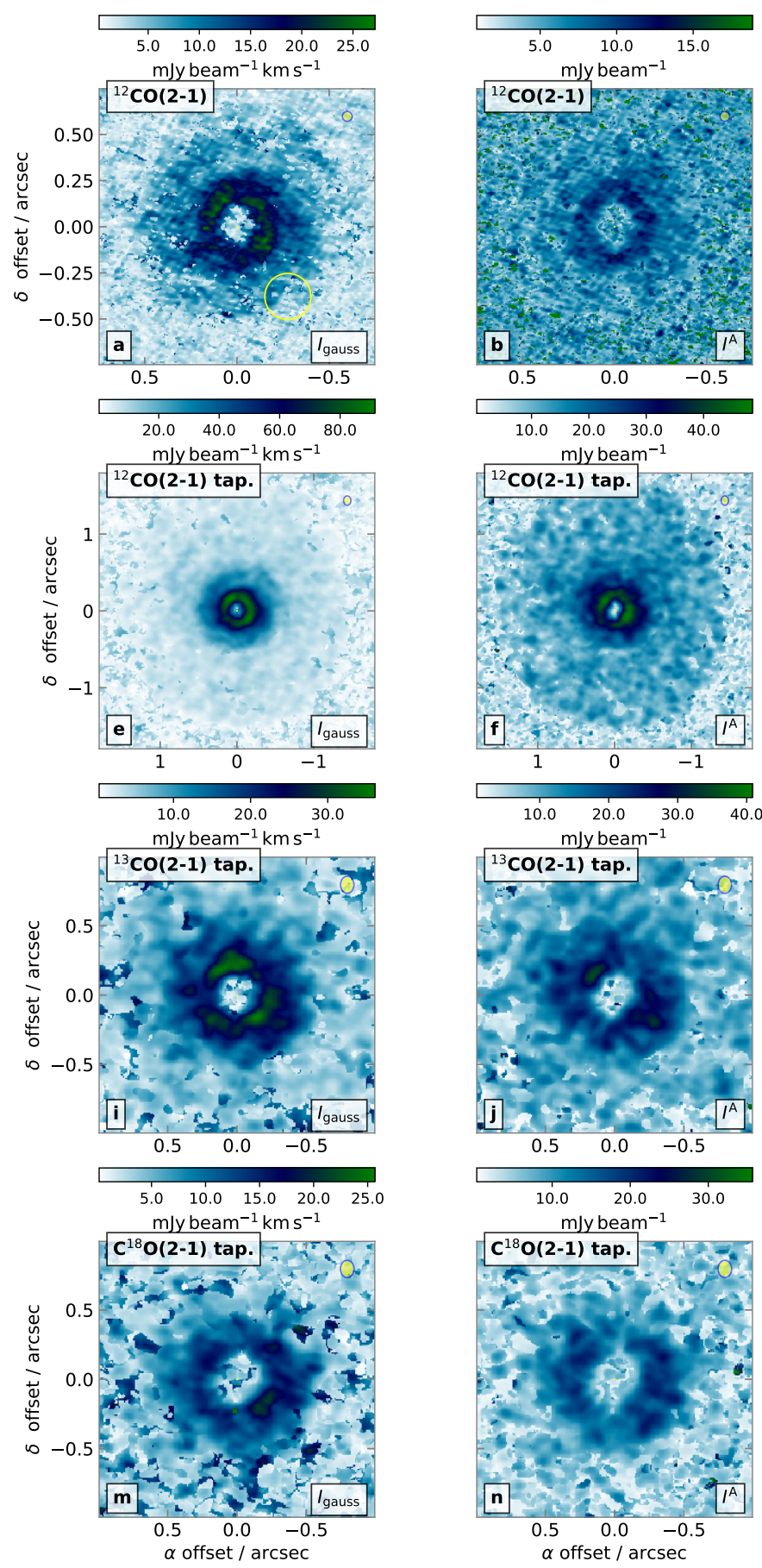
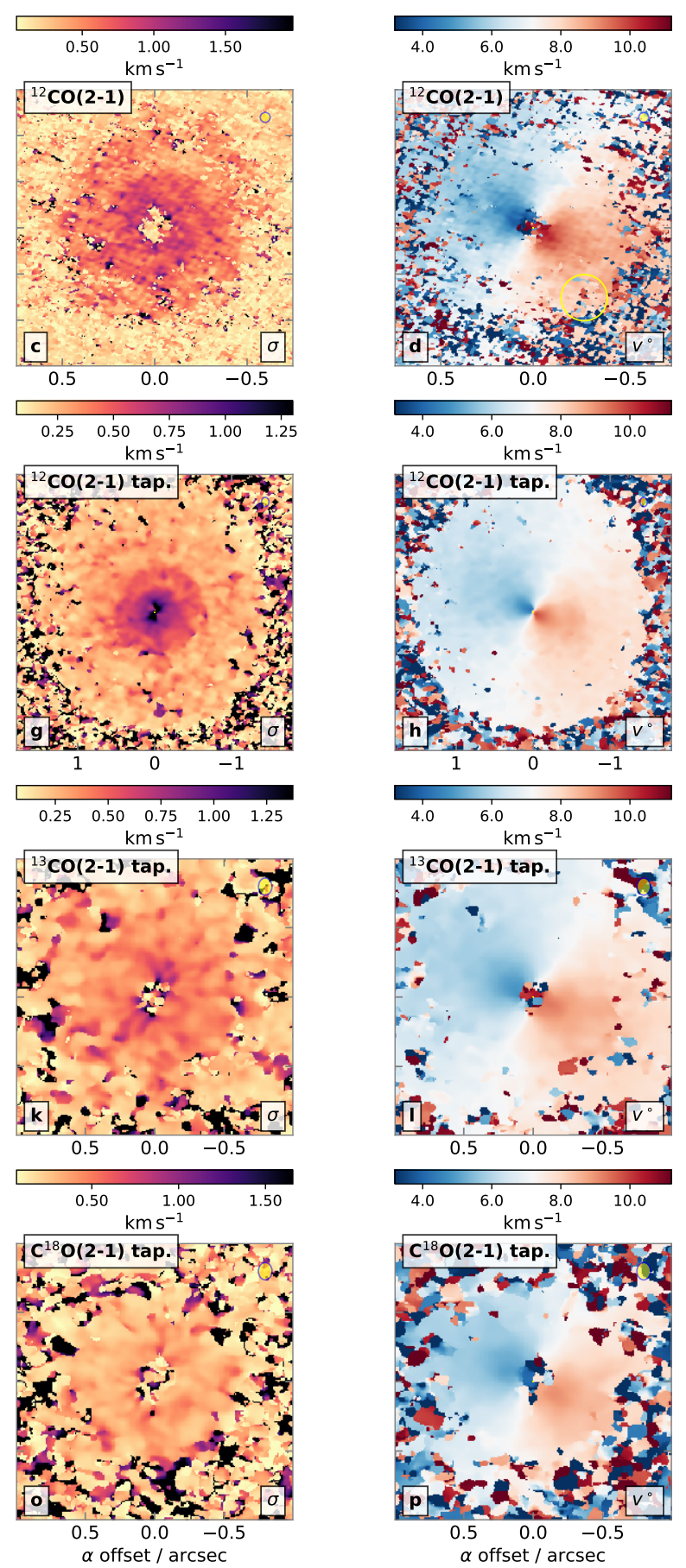

Figure 2. Moment maps in ${ }^{12} \mathrm{CO}(2-1),{ }^{13} \mathrm{CO}(2-1)$ and $\mathrm{C}^{18} \mathrm{O}(2-1)$ extracted from the tclean datacube using single-Gaussian fits in velocity. The yellow circle marks the same region of interest as in Fig. 1. The first row shows, for ${ }^{12} \mathrm{CO}(2-1)$, a) the Gaussian velocity-integrated intensity, b) the Gaussian amplitude, c) the Gaussian dispersion, and d) the Gaussian velocity centroid. The beam $\left(00^{\prime \prime} 054 \times 00^{\prime \prime} 040 / 89.4 \mathrm{deg}\right)$ is indicated by a yellow ellipse. The second to fourth rows extend the same images to tapered versions of ${ }^{12} \mathrm{CO}(2-1)\left(0\right.$. . $121 \times 00^{\prime \prime} 097 /-87$ deg beam $),{ }^{13} \mathrm{CO}(2-1)\left(0{ }^{\prime \prime} 121 \times 0\right.$.'097 / -88 deg beam $)$, and $\mathrm{C}^{18} \mathrm{O}(2-1)$ $(0$ " $125 \times 0$ '” $098 /-88$ deg beam). Note the larger field of view for e)-h).

servations, respectively. The traces inferred with this procedure are shown as cyan crosses in Fig. 6a. We then fitted these traces to the equation of a general Archimedean spiral $\left(r=a+b \theta^{n}\right)$, and found the best-fit parameters using SCIPY's Nelder-Mead minimisation algorithm. Since the objective of this fit is the comparison of the $\mathrm{CO}$ spirals with the IR spirals, we did not deproject the disc, hence the choice for a general Archimedean spiral equation for the fit.

We then proceeded to a similar analysis in the 2016-06-30 near-IR polarised intensity image of the system obtained with SPHERE/IRDIS (Stolker et al. 2017). The southwest spiral shows a sudden discontinuity in pitch angle, identified as 'kink' in Muto 

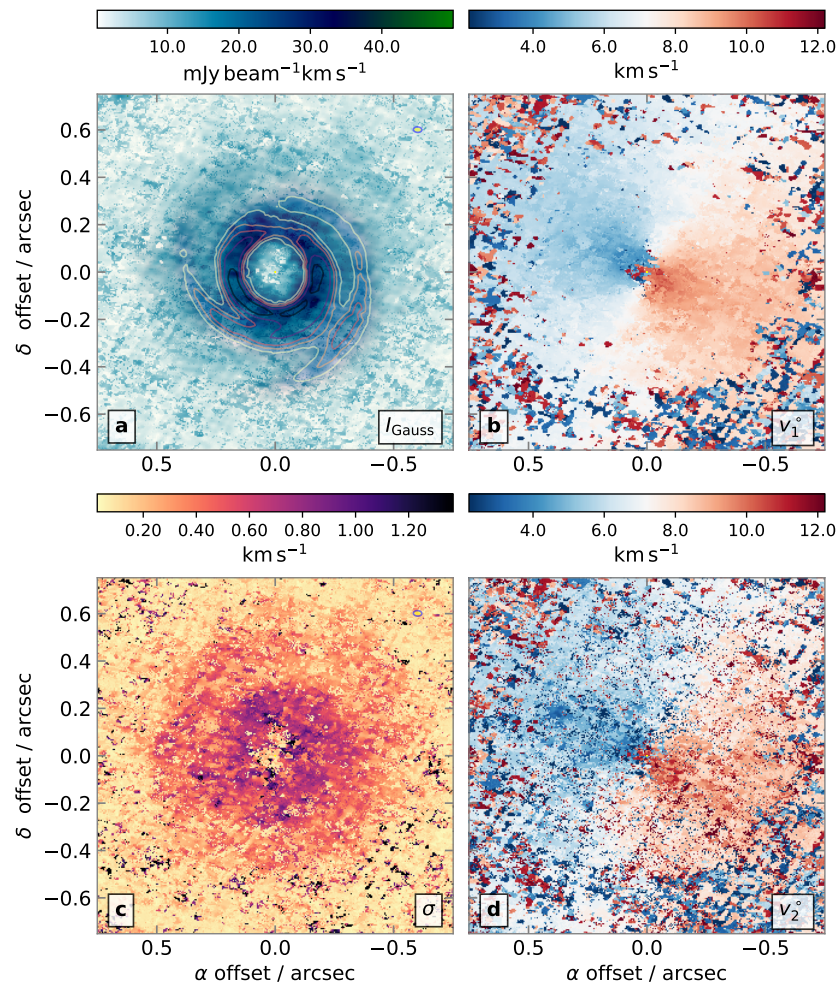

Figure 3. Moment maps in ${ }^{12} \mathrm{CO}(2-1)$ extracted from the UvMEM-restored datacube using double-Gaussian fits in velocity, and comparison with the $H$-band polarised intensity. a) The 2-Gaussian velocity-integrated intensity in colour scale, compared with the $H$-band $Q_{\phi}$ image from Fig. 4, traced with the same contours. b) The velocity centroid of the brighter Gaussian. c) The Gaussian dispersion. d) The velocity centroid of the fainter Gaussian.

et al. (2012) and Stolker et al. (2016). They attributed the kink to the location of a planet driving this spiral arm, which they labelled ' $\mathrm{S} 1$ '. Therefore we adopted a different equation for the fit of this specific spiral trace. Instead of a general Archimedean spiral, we considered the expected shape from a spiral density wave excited by an embedded planet in the linear or weakly non-linear regime (Rafikov 2002):

$$
\begin{aligned}
\theta(r) & =\theta_{p}+\frac{\operatorname{sign}\left(r-r_{p}\right)}{h_{p}} \\
& \times\left\{\left(\frac{r}{r_{p}}\right)^{1+\beta}\left[\frac{1}{1+\beta}-\frac{1}{1-\alpha+\beta}\left(\frac{r}{r_{p}}\right)^{-\alpha}\right]\right. \\
& \left.-\left(\frac{1}{1+\beta}-\frac{1}{1-\alpha+\beta}\right)\right\}
\end{aligned}
$$

where $\left(\theta_{p}, r_{p}\right)$ are the polar coordinates of the planet location, $h_{p}$ is the disc aspect ratio at the planet's location, and $\alpha$ and $\beta$ are the exponents of power laws for the angular frequency of the disc $\left(\Omega \propto r^{-\alpha}\right)$ and the sound speed $\left(c_{s} \propto r^{-\beta}\right)$, respectively. The five parameters of this equation are known to be highly degenerate (e.g. Muto et al. 2012; Christiaens et al. 2014). Furthermore, the linear or weakly non-linear approximation is only valid in the vicinity of the planet (e.g. Zhu et al. 2015), with an increasingly larger pitch angle (with respect to the linear approximation) the further the separation with the planet location. Given these considerations, we restricted the spiral trace to only a section subtending $45 \mathrm{deg}$ around the twist.

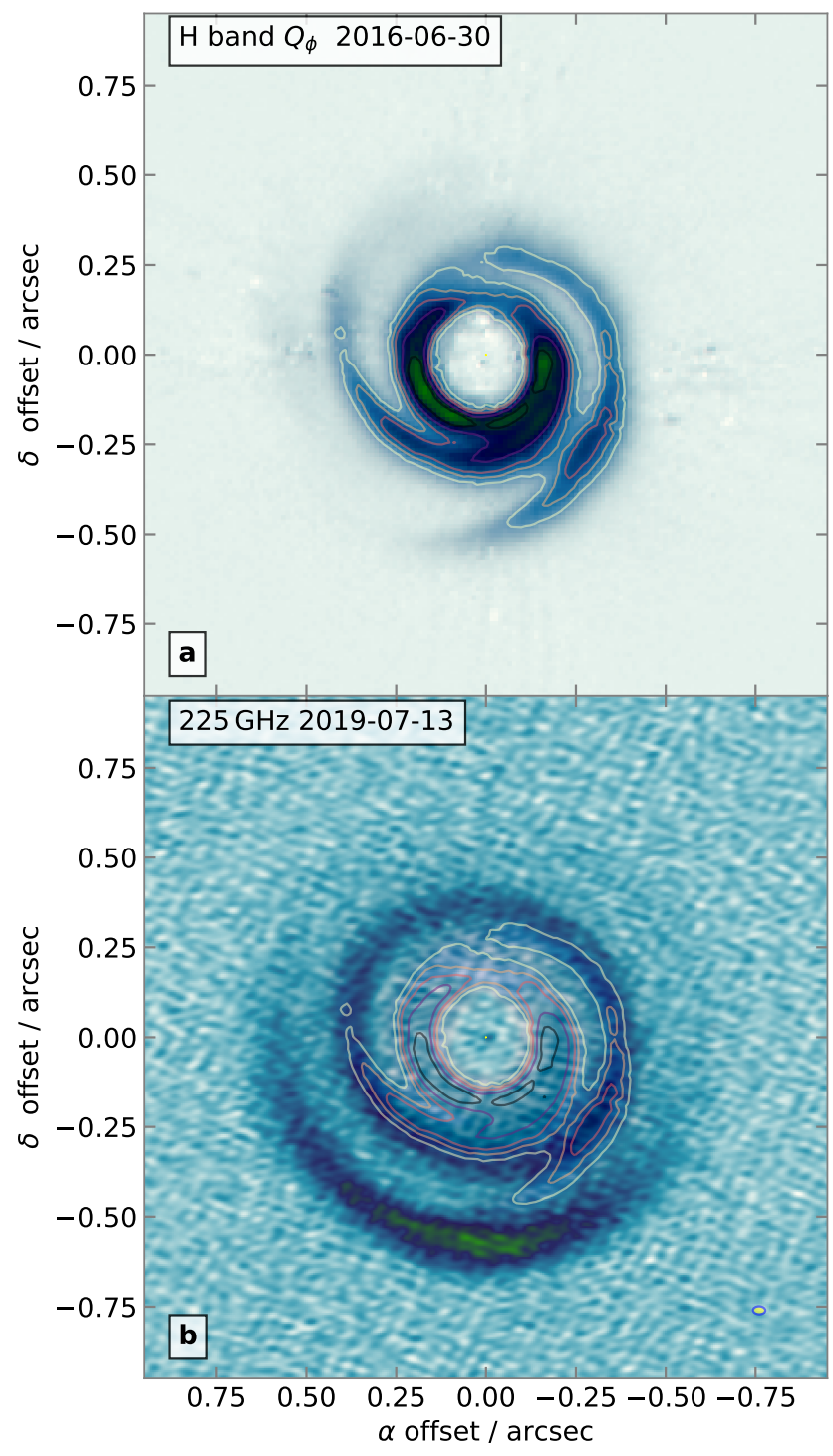

Figure 4. a: IRDAP reduction of the H-band $Q_{\phi}$ image data from Stolker et al. (2017), with contours taken at peak-intensity fractions of 0.2, 0.3, 0.4, $0.6,0.8 . \mathbf{b}$ : Overlay of the same contours as in a) on the Briggs $r=0$ image from Fig. 1b.

Moreover, we fixed $\alpha$ to 1.5 (Keplerian rotation) and $\beta$ to 0.207 , the average flaring found in Avenhaus et al. (2018) based on their sample of $H$-band polarised intensity images of protoplanetary discs, and left $\theta_{p}, r_{p}$ and $h_{p}$ as free parameters. Since Eq. 3 assumes a face-on view of the disc, we deprojected the near-IR image using DISKMAP ${ }^{3}$ (Stolker et al. 2016), adopting $\beta=0.207$ and an initial estimate of 0.1 for $h_{p}$. We also scaled the image by $r^{2}$ to compensate for stellocentric flux dilution. We then proceeded with finding the optimal values of $\theta_{p}, r_{p}$ and $h_{p}$ with scipy's Nelder-Mead minimisation algorithm, iteratively updating the value of $h_{p}$ used for the disc deprojection. The values of $h_{p}$ used for deprojection and found by the fit of the spiral traces converged to within $0.1 \%$ relative values within 2 iterations. The final values we inferred are a PA of $212.4 \pm 0.7 \mathrm{deg}$ and $r=425.9 \pm 1.2 \mathrm{mas}$

\footnotetext{
${ }^{3}$ https://github.com/tomasstolker/diskmap
} 

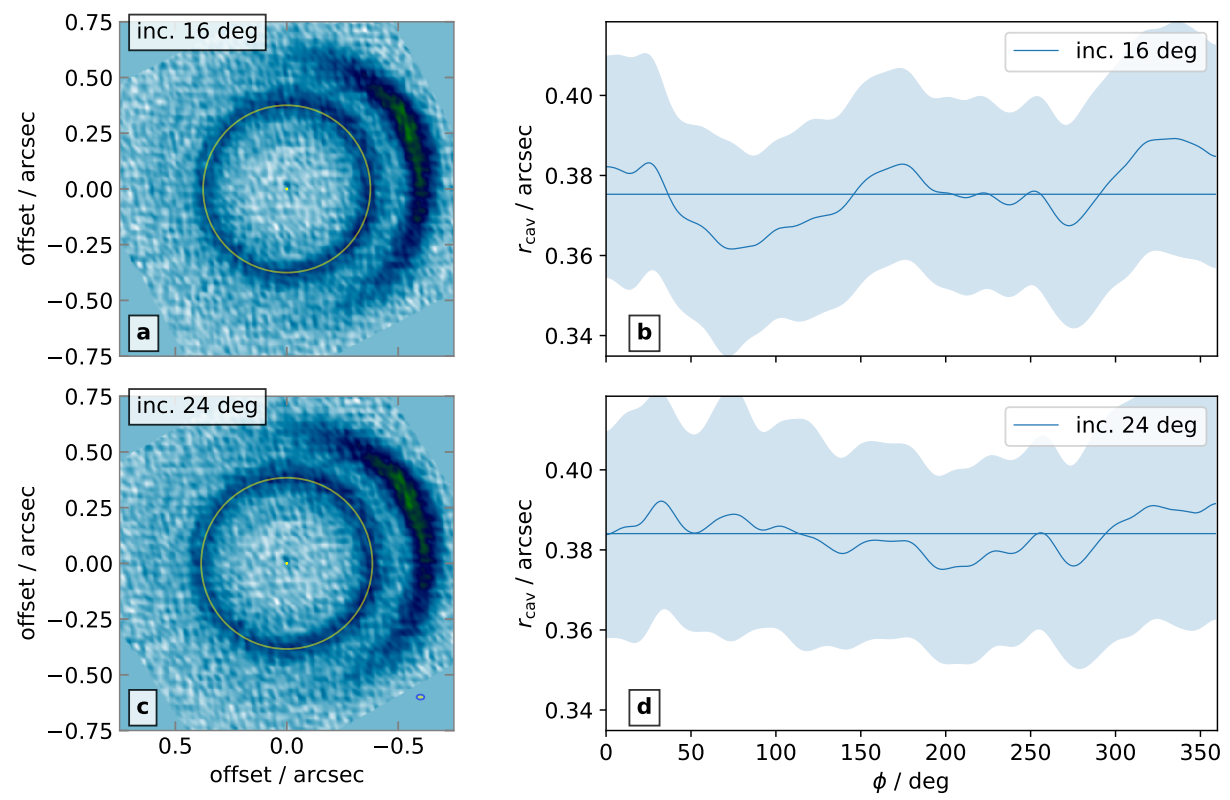

Figure 5. a: face-on view of the Briggs $r=0$ image from Fig. 1b, assuming an inclination of $16 \mathrm{deg}$. b: the azimuthal profile of the Gaussian centroid and dispersion in the inner ring, in a polar expansion of the image in a), after smoothing over two beam major axis (the total height of the shaded area corresponds to $1 \sigma$ ). c: and d: same as a) and b), for the optimal orientation of $24 \mathrm{deg}$.
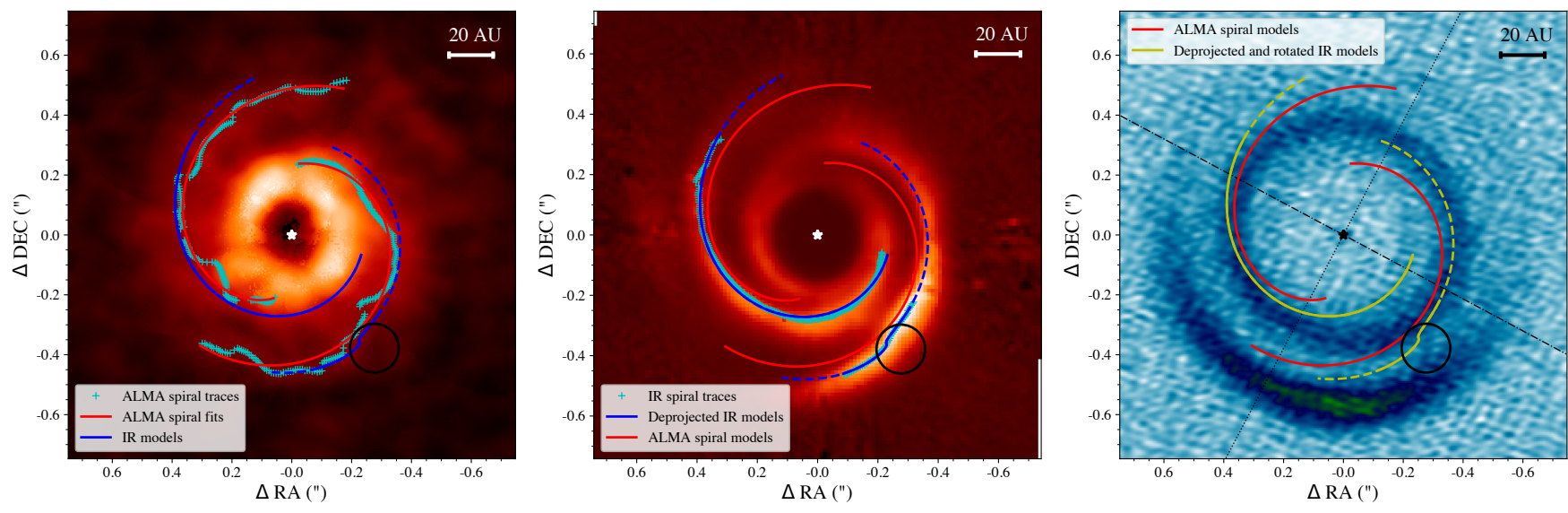

Figure 6. Spiral traces identified in the ${ }^{12} \mathrm{CO}(2-1)$ moment 0 map and the near-IR scattered-light image (cyan crosses), and corresponding best-fit spiral models compared to the ${ }^{12} \mathrm{CO}(2-1)$ moment 0 map (left), the deprojected near-IR scattered-light image (middle) and the sub-mm continuum image (right). The location of the gap-crossing sub-mm continuum filament is circled in all panels. The IR spiral models are shown projected (i.e. as observed) in the left panel, and deprojected onto the plane of the sky (i.e. after flaring and inclination correction) in the middle panel. In the sub-mm continuum image, the deprojected model for the south-west IR spiral is rotated by $3.7 \mathrm{deg}$ in the prograde direction, and subsequently projected onto the disc mid-plane (i.e. considering disc inclination, but not surface flaring). The semi-major and semi-minor axes are shown with dotted-dashed and dotted lines respectively. See text for details.

$(57.8 \pm 0.2 \mathrm{au})$ for the planet's location, and a disc aspect ratio $h_{p}=$ $0.017 \pm 0.001$ at that location, at the epoch of the IRDIS observations. The uncertainties on each parameter were found by bootstrapping (1000 bootstraps). We tested different values of $\beta$ ranging from 0 to 0.25 and found consistent values of $\theta_{p}, r_{p}$ and $h_{p}$. Fig. $6 \mathrm{~b}$ shows the best-fit model onto the deprojected disc image, with intensities scaled by $r^{2}$. We notice a tentative radial shift between the $\mathrm{CO}$ models and IR spirals, which is likely due to the different emitting and scattering surfaces for ${ }^{12} \mathrm{CO}(2-1)$ and sub- $\mu \mathrm{m}$ size dust grains, respectively (e.g. Pinte et al. 2018; Avenhaus et al. 2018).

Finally, we compared all spiral models to our sub-mm continuum image of the disc (Fig. 6c). Taking into account Keplerian rotation around a star with mass $M_{\star}=1.67_{-0.16}^{+0.18} M_{\odot}$ (Wichittanakom et al. 2020), and for a distance of $135.7 \pm 1.4 \mathrm{pc}$ (Gaia Collaboration et al. 2018), the difference of $3.04 \mathrm{yrs}$ between the epochs of the IRDIS and ALMA data corresponds to a prograde rotation of $\sim 3.7 \mathrm{deg}$. The IR model for S1 is thus rotated by this amount in the right panel of Fig. 6. More precisely, we used the deprojected IR model (shown in the middle panel of Fig. 6) for the rotation and subsequent reprojection onto the disc mid-plane (i.e. considering the inclination of the disc, but not the original flaring) - see left panel of Fig. 6 for the relative locations of the non-deprojected spiral model with 
respect to the filament. This angular shift of $3.7 \mathrm{deg}$ nicely aligns the PA of the tentative filament $(\sim 216.1 \mathrm{deg}$; Sect. 3.1) and that of the IR spiral arm twist $(216.1 \pm 0.7 \mathrm{deg}$ after rotation). However, we measure $\mathrm{a} \gtrsim 42.1 \pm 1.2$ mas radial shift between the centre of the filament ( 468mas) and the planet location inferred from our fit to the IR twist, even after deprojection. The imprecision in the location of the star in the ALMA image appears insufficient to account for this radial shift, as it is $\lesssim 15$ mas, e.g. considering the brightest sub-mm continuum signal in the cavity is tracing the star (see inset of Fig. 1 a). The observed radial shift may be consistent with the expected curling of the spiral shock towards the star (e.g. Zhu et al. 2015, more details in Sec. 4) as it would imply the IR scattering surface to be located at a shorter deprojected radius than the bulk of the density wave (likely consistent with the continuum filament locus). It is worth noting that given the likely eccentric geometry of the inner ring (Sec. 3.1), the planet may also be on a slightly eccentric orbit, which may also partially contribute to this offset.

\subsection{Line diagnostics of physical conditions}

The uniform slab approximation goes a long way in observational astronomy, as it is a simple means to extract physical conditions in a given line-of-sight. We use this approximation to estimate physical conditions in the gas using the $\mathrm{CO}$ isotopologue rotational lines. In local-thermodynamic-equilibrium (LTE) the emergent intensity from ground-state rotational lines depends on the column of the emitting specie, on the uniform-slab temperature $T_{b}$, and on the line-of-sight turbulent broadening $v_{\text {turb }}$. We write the gas temperature with an under-script ' $b$ ' to remind that, in the case of $\mathrm{CO}$, this temperature is close to the brightness temperature of the optically thick ${ }^{12} \mathrm{CO}$. Given fractional abundances, the emitting column can be converted into a total gas surface density, $\Sigma_{g}$. We have developed a tool to fit multi-isotopologue data with these free parameters, which we call SLAB.Line. Related approaches have also been considered by others (Teague et al. 2016; Flaherty et al. 2020; Garg et al. 2020; Yen \& Gu 2020). The model line profile for a given line of sight $\vec{x}$ is

$I_{v}^{m}(\vec{x})=B_{v}\left(T_{b}(\vec{x})\right)\left[1-\exp \left(-\tau_{v}(\vec{x})\right)\right]$,

as a function of frequency $v$, with

$\tau_{v}(\vec{x})=\kappa_{L}(\vec{x}) \Sigma_{g}(\vec{x}) f_{\mathrm{mol}} \Phi_{v}(\vec{x})$.

The line opacity $\kappa_{L}(\vec{x})$ is approximated in LTE, so for a rotational transition $J_{2} \rightarrow J_{1}$

$\kappa_{L}(\vec{x})=\frac{h \nu_{\circ}}{4 \pi m_{\mathrm{H}_{2}}} \frac{g_{J_{1}} e^{-\frac{E_{J_{1}}}{k T_{b}(\vec{x})}}}{Z(\vec{x})} B_{12}\left[1-e^{-\frac{h \nu_{\circ}}{k T_{b}(\vec{x})}}\right]$.

We use the LAMDA molecular database (Schöier et al. 2005), and evaluate the partition function $Z=\sum_{J=0}^{J_{\max }} g_{J} e^{-\frac{E_{J}}{k T_{b}}}$ by summing over all tabulated rotational energy levels $E_{J}$ (so for ${ }^{12} \mathrm{CO}$ this corresponds to $J_{\max }=40$ ). In Eq. $5, f_{\operatorname{mol}}$ is the abundance by number of the emitting molecule relative to $\mathrm{H}_{2}$. In this case for the $\mathrm{CO}$ isotopologues we set $f_{13} \mathrm{CO}=\frac{1}{76} f_{12} \mathrm{CO}$ (from Casassus et al. 2005; Stahl et al. 2008), $f_{\mathrm{C}^{18} \mathrm{O}}=\frac{1}{500} f^{12} \mathrm{CO}$ (Wilson \& Rood 1994), with $f_{12} \mathrm{CO}=10^{-4}$. The line profile is simply a thermal Gaussian broadened by turbulence, with dispersion $v_{\text {turb }}(\vec{x})$ and velocity centroid $v_{\circ}(\vec{x})$ :

$\Phi_{v}(\vec{x})=\frac{1}{\sqrt{2 \pi} \sigma_{v_{\circ}}} \exp \left(-\frac{\left(v-v_{\circ}\right)^{2}}{2 \sigma_{v_{\circ}}^{2}}\right)$, with $v_{\circ}=\frac{E_{J_{2}}-E_{J_{1}}}{h}\left(1+\frac{v_{\circ}}{c}\right)$ and

$\sigma_{v_{\circ}}(\vec{x})=\frac{v_{\circ}}{c} \sqrt{\frac{k T_{b}(\vec{x})}{m_{\text {mol }}}+v_{\text {turb }}^{2}(\vec{x})}$,

and where $m_{\mathrm{mol}}$ is the molecular mass.

The free parameters $v_{\circ}, \Sigma_{g}, T_{b}$ and $v_{\text {turb }}$ were constrained in each line of sight $\vec{x}_{l}$ independently with a least-squares fit to the observed spectra in the three isotopologue transitions:

$\chi^{2}\left(\vec{x}_{l}\right)=\sum_{i=1}^{3} \frac{1}{I_{\mathrm{rms}, \mathrm{i}}^{2}\left(\vec{x}_{l}\right)} \sum_{v_{k}}\left(I_{v_{k}}\left(\vec{x}_{l}\right)-I_{v_{k}}\left(\vec{x}_{l}\right)^{m}\right)^{2}$,

where the sum in frequencies $\left\{v_{k}\right\}$ runs over all available spectral channels. The noise in each line of sight, $I_{\mathrm{rms}, i}^{2}\left(\vec{x}_{l}\right)$, is taken as the rms dispersion in the observed spectra $\pm 1 \mathrm{~km} \mathrm{~s}^{-1}$ away from the peak of the line. An application to HD 135344B is shown in Fig. 7, where we report the inferred fields for $\Sigma_{g}(\vec{x}), T_{b}(\vec{x})$ and $v_{\text {turb }}(\vec{x})$. It is particularly interesting to note the similarity between $v_{\text {turb }}$ and the line velocity dispersion map $\sigma(\vec{x})$ in Figs. 2 and 3. These fits assumed fixed isotopologue abundances, but we reach thermal residuals nonetheless. Example fits are shown in Figs. 8.

From the maps in Fig. 7 we note that $\mathrm{CO}$ line temperatures are in excess of $115 \mathrm{~K}$ (which would be the dust temperature for water condensation) out to $\sim 25 \mathrm{au}$. Within $\sim 20 \mathrm{au}$, confusion of different Keplerian velocities in the finite beam exaggerate the turbulent velocities along an inner ring inset within the hot inner ring seen in $T_{b}$, which is itself inset within the dense ring seen in $\Sigma_{g}$. The lack of signal inside the ${ }^{12} \mathrm{CO}$ central cavity (within $0 .{ }^{\prime \prime} 1$ ) yields spuriously low values for $T_{b}$.

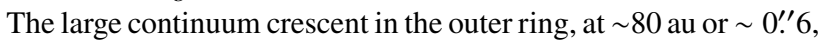
is absent from the $\Sigma_{g}(\vec{x})$ map in Fig. 7 (and also from the azimuthal averages in Fig. 9). This may seem surprising in the context of the dust trap interpretation, since the required local pressure maximum should correspond to a local peak in $\Sigma_{g}$ by a factor of a few relative to the azimuthal average (Birnstiel et al. 2013). However, a similar result is seen in MWC 758, where the position of the dust peak does not correspond to a maximum in $\Sigma_{g}$, as inferred from the CO isotopologues(Boehler et al. 2018). In HD 135344B, while it appears that the $\mathrm{CO}$ mass surface density is fairly homogeneous across the disc, it may be that the $\mathrm{CO}(2-1)$ isotopologues come short of reaching deep enough to sample the total mass surface density. This could result from freezing of $\mathrm{CO}$ onto dust grains, or because the continuum is optically thick, so that thermal equilibrium between dust and gas in the denser regions would leave no net line emission after continuum subtraction (see Boehler et al. 2017, for a detailed description of the effect).

The radial profiles for $\Sigma_{g}$ and $T_{b}$, obtained with azimuthal averages and a disc inclination of $i=17.6 \mathrm{deg}$, are shown in Figs. $9 \mathrm{~b}$ and $9 \mathrm{c}$. The best fit values for $T_{b}$ come close, at $\sim-30 \%$, of the radial temperature profile of the $\tau=1$ surface in the ${ }^{12} \mathrm{CO}(2-1)$ line as estimated from detailed thermochemical modelling of the (3-2) $\mathrm{CO}$ isotopologue lines (DALI model by van der Marel et al. 2016a). In Fig. $9 \mathrm{c}, T_{b}$ appears to be above the $\mathrm{CO}$ sublimation temperature of $\sim 20 \mathrm{~K}$ everywhere in the $\mathrm{CO}$ layer. However, colder CO in the midplane could have condensed on the dust grains, where the gas phase $\mathrm{CO}$ abundance is much lower, $f_{12} \mathrm{CO} \sim 10^{-12}$ van der Marel et al. (2016a). This is reflected in the factor of $\sim 10$ larger gas surface density derived from the $\mathrm{CO}(3-2)$ isotopologues with DALI, as shown in Fig. 9b. The SLAB.Line diagnostics thus yield a lower limit to the total gas mass, since in general $f_{12} \mathrm{CO}<10^{-4}$. The general structure of $\Sigma_{g}(R)$ inferred from SLAB.Line is consistent with previous estimates based on parametric modelling, either with the location of the 

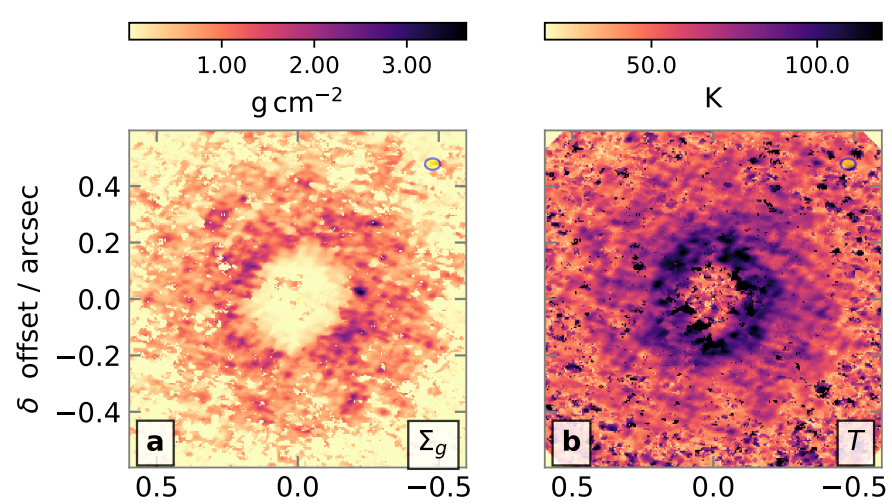
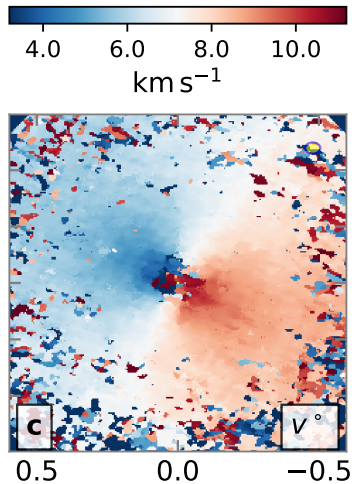
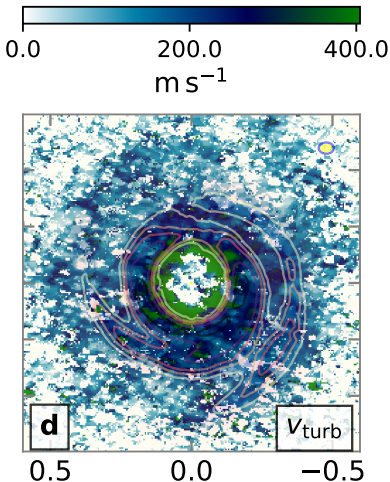

Figure 7. Example application of SLAB.Line to extract physical conditions in LTE using the ${ }^{12} \mathrm{CO}(2-1),{ }^{13} \mathrm{CO}(2-1)$ and $\mathrm{C}^{18} \mathrm{O}(2-1)$ lines: a): total gas surface density $\Sigma_{g}, \mathbf{b}$ ): gas temperature $T_{b}, \mathbf{c}$ ): centroid velocity $v_{\circ}, \mathbf{d}$ ): turbulent line broadening $v_{\text {turb. }}$ The contours tracing the $H$-band $Q_{\phi}$ image from Fig. 4 are overlaid on d) to compare with the near-IR spiral pattern.
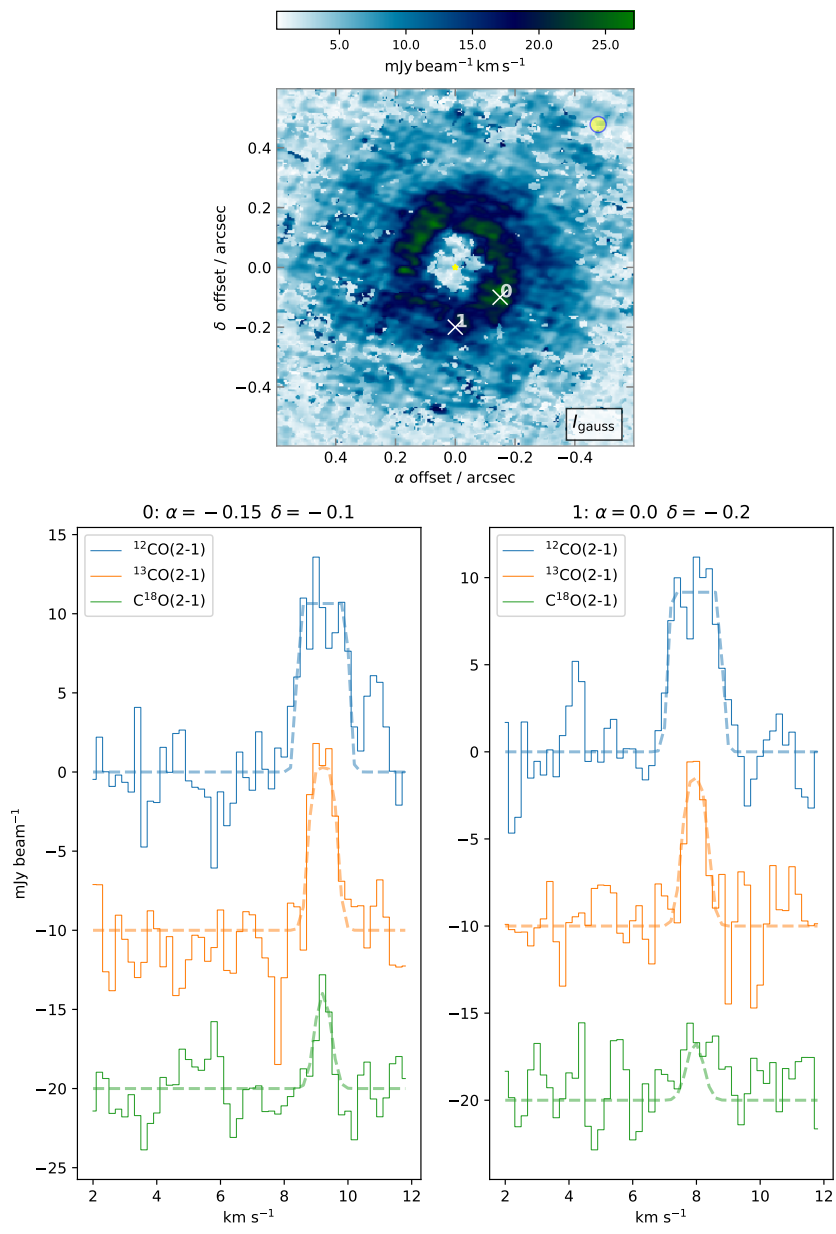

Figure 8. Comparison of observed and best fit SLAB.LinE spectra for selected lines of sights, at the offset R.A. $(\alpha)$ and Dec. $(\delta)$ reported as titles to the spectral profiles, and also shown in the image at the top. The observed spectra are drawn in solid lines, while the model is shown in thick dashed lines. sharp gas cavity edge placed at 30 au by van der Marel et al. (2016a), or with the gradual density drop inwards used by van der Marel et al. (2021), with a gap at $\sim 20$ au.

The lack of ${ }^{12} \mathrm{CO}$ integrated intensity at the centre of the cavity is suggestive of a very low column density of ${ }^{12} \mathrm{CO}$, and is reflected in the very low value of $\Sigma_{g}$ near the star, which is consistent with zero. This central hole could be caused by photo-dissociation driven by UV irradiation. The ${ }^{12} \mathrm{CO}$ integrated intensity is so low in the disc's innermost regions that photo-dissociation could act down to the disc mid-plane. For this to happen, gas surface densities $\lesssim 10^{-2}$ $\mathrm{g} \mathrm{cm}^{-2}$ are necessary (see, e.g., Eq. 4 of Baruteau et al. 2021 for $z=0$ ). Interestingly, the modelling of $\mathrm{CO}$ ro-vibrational observations by Carmona et al. (2014) indicates that the gas surface density in the cavity of the HD135344B should be $\lesssim 10^{-2} \mathrm{~g} \mathrm{~cm}^{-2}$ (see the lower-right panel in their Fig. 6), which would support the idea that photo-dissociation could indeed be responsible for the lack of ${ }^{12} \mathrm{CO}$ integrated intensity inside the cavity.

\subsection{Rotation curve}

The rotation curve can be extracted from the velocity centroid map and brings constraints on the central (stellar) mass and orientation (Casassus \& Pérez 2019). From the observations presented in Sec. 2 , the velocity field of the top layer in ${ }^{12} \mathrm{CO}(2-1)$ is best traced with the double-Gaussian moments applied to either the UVMEM-restored or the $u v$-tapered tclean data cubes. For conciseness we choose to report on the $u v$-tapered version, as it allows an extension to larger radii and is consistent with the results from the finer angular resolutions, even at radii as small as $0 .{ }^{\prime \prime} 25$.

The 3 -D rotation curve $\overrightarrow{\tilde{v}}(R)=\left(\tilde{v}_{R}(R), \tilde{v}_{\phi}(R), \tilde{v}_{z}(R)\right)$, in disccentred cylindrical coordinates where $z=0$ coincides with the midplane, also informs on large scale radial and vertical flows (Teague et al. 2019). We extended the same procedure as described in Casassus \& Pérez (2019) to 3-D in the ConeRot package, publicly available at https://github.com/simoncasassus/ConeRot. In an axially symmetric disc the unit opacity surface in an optically thick line such as ${ }^{12} \mathrm{CO}(2-1)$ can be represented by its height over the mid-plane, $H_{1}(R)$. We approximate this surface by a series of cones whose orientations are fit to the observed velocity centroid in concentric radial domains, or 'regions', which we combine by averaging as described in Casassus \& Pérez (2019). The procedure is similar to that followed by the EDDY package (Teague et al. 2019), except that the disc ori- 


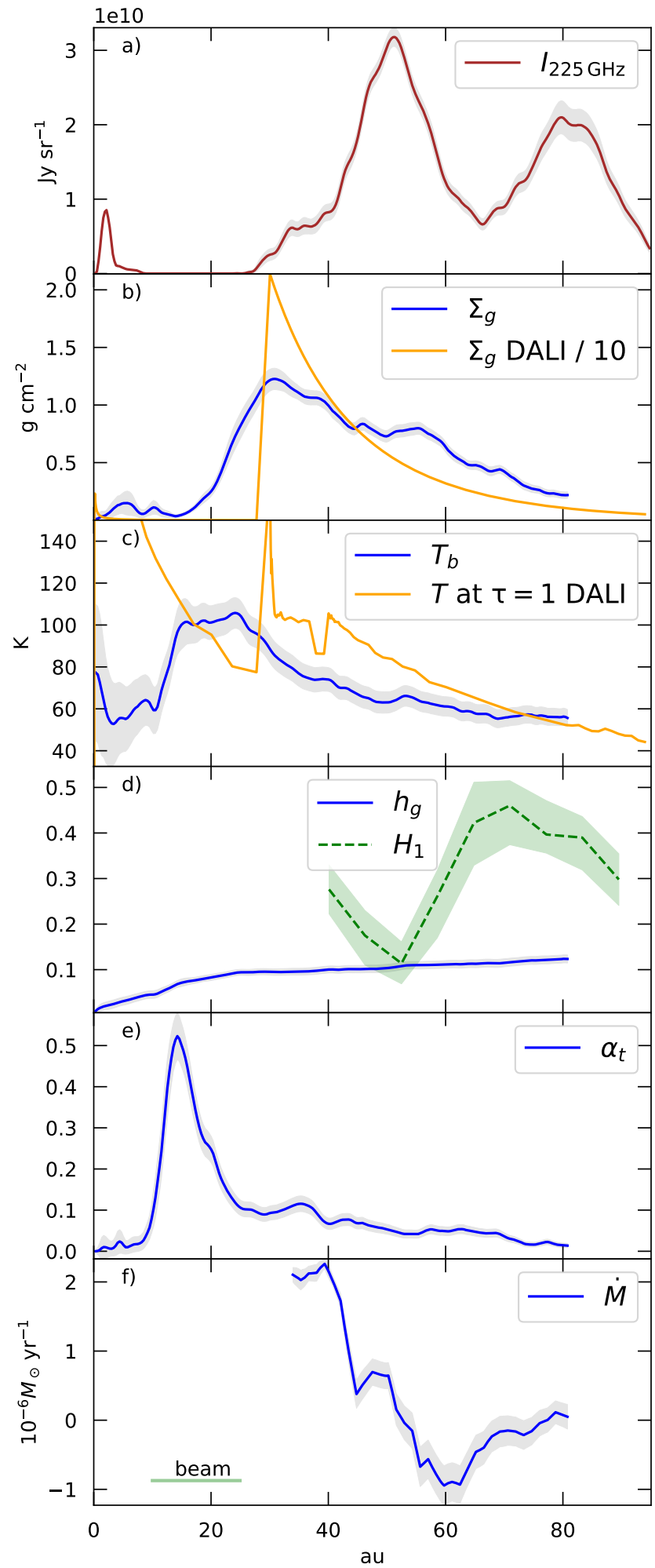

Figure 9. Radial continuum intensity profile and properties of the HD 135344B inferred from the uniform slab diagnostics with SLAB.Line and from the disc rotation curve: a) Azimuthal average of the $225 \mathrm{GHz}$ continuum intensity profile extracted from the image in Fig. 1c. b) gas surface density profile $\Sigma_{g}(R)$, c) gas temperature profile $T_{b}(R)$, d) aspect ratio profile $h_{g}(R)=c_{s} / \Omega_{K}$, and comparison with the unit-optical-depth surface $H_{1}(R)$, e) turbulence parameter $\alpha_{t}=\left(v_{\text {turb }} / c_{S}\right)^{2}$, f) accretion rate $\dot{M}(R)=-2 \pi R \Sigma_{g} \tilde{v}_{R}$. entation along with the rotation curve are both optimised to fit $v_{1}^{\circ}$ in each region.

A full optimisation to fit $v_{1}^{\circ}$ over the radial domain $\left[0,{ }^{\prime \prime} 3,0\right.$.' 7$]$, with an axially symmetric model in a purely azimuthal flow, results in $i=17.6_{-3.2}^{+2.7} \mathrm{deg}, \mathrm{PA}=241.9_{-0.5}^{+0.4} \mathrm{deg}$, and an aspect ratio $h_{1}=$ $0.28_{-0.05}^{+0.06}$ (for the unit-opacity surface). Compared to the scale height inferred in Sec. $4, h_{1} \sim 3 h$. The systemic velocity we measure is $v_{\text {lsr }}=7.12 \pm 0.02 \mathrm{~km} \mathrm{~s}^{-1}$ (see Casassus \& Pérez 2019, for details). We then fixed PA and $i$, and proceeded to optimise the aspect ratio and the rotation curve in 11 radial bins over [0.'25, 0.'75], which produced the 3-D rotation curve shown in Fig. 10. A face-on view of the disc using this geometry is shown in Fig. 11. The deviations from an axially flow appear to be thermal, although more pronounced in the region around the possible filament seen in the continuum. Deeper data are required to discuss these velocity deviations.

The dynamical stellar mass, fit to the tangential component $\tilde{v}_{\phi}(R)$, is $1.59 \pm 0.04<M_{\star} / M_{\odot}<1.81 \pm 0.04$. The lower limit stems from assuming perfect cylindrical rotation, while the upper limit corresponds to Keplerian vertical shear. This stellar mass is consistent with $M_{\star}=1.67_{-0.16}^{+0.18} M_{\odot}$, as measured from the photospheric spectrum (Wichittanakom et al. 2020). For comparison, if we fix $i=24 \mathrm{deg}$ (as inferred from the continuum), then $0.91 \pm 0.04<M_{\star} / M_{\odot}<$ $0.98 \pm 0.04$.

\section{DISCUSSION}

If the sub-mm continuum filament traces a planetary wake, one may expect the planet in the sub-mm annular gap to also carve a dip in the gas surface density, whose signature may be observable in ${ }^{12} \mathrm{CO}$ if the planet is massive enough. The last panel of Fig. 10 shows a dip in the opening angle of the cone tracing the ${ }^{12} \mathrm{CO}(2-1)$ unit opacity surface, corresponding to an aspect ratio of $\sim 0.1$ at $\sim 0$.' 4 separation (i.e. in the sub-mm annular gap), compared to $h \gtrsim 0.2$ beyond the annular gap.

We showed in Sec. 3.2 that the sub-mm continuum gap-crossing filament was almost coincident with a twist in the southwest spiral seen at near-IR wavelengths. We found the best-fit disc aspect ratio for the scattering surface to be $0.017 \pm 0.001$. This value appears significantly smaller than the expected value based on the ${ }^{12} C O(2-1)$ brightness temperature though ( $h \sim 0.09$; Fig. 9). One possibility to account for both the very low inferred value of $h_{p}$ and the apparent radial shift between the IR twist in the trace of S1 and the filament (Sec. 3.2) is that the outer spiral arm (with respect to the twist) is more curled in towards the star at the $H$-band scattering surface than the inner spiral (as e.g the 3D simulations in Zhu et al. 2015). Since the value of $h_{p}$ inferred in the fit depends on the apparent radial amplitude of the twist, a more curled in outer spiral would artificially reduce the inferred value of $h_{p}$. Furthermore, this curling-in would also explain the radial shift between the outer part of S1 and the azimuthal asymmetry in the outer sub-mm continuum ring. The latter appears to be located in the continuity of the filament, and it may thus be tracing dust trapping induced by the density enhancement in the planetary wake.

As noted in Sec.2, the same 2-armed grand-design spiral that characterises the near-IR scattered-light images also stands out in the ${ }^{12} \mathrm{CO}(2-1)$ velocity-integrated intensity and in the line velocity dispersion (or second-order moment). However, the spiral pattern is absent in the peak intensity map (Fig. 2). The absence of a counterpart in peak intensity suggests that the spiral structure is not due to a local enhancement in temperature, but is instead due either to a strong modulation in gas surface density, or to enhanced velocity 


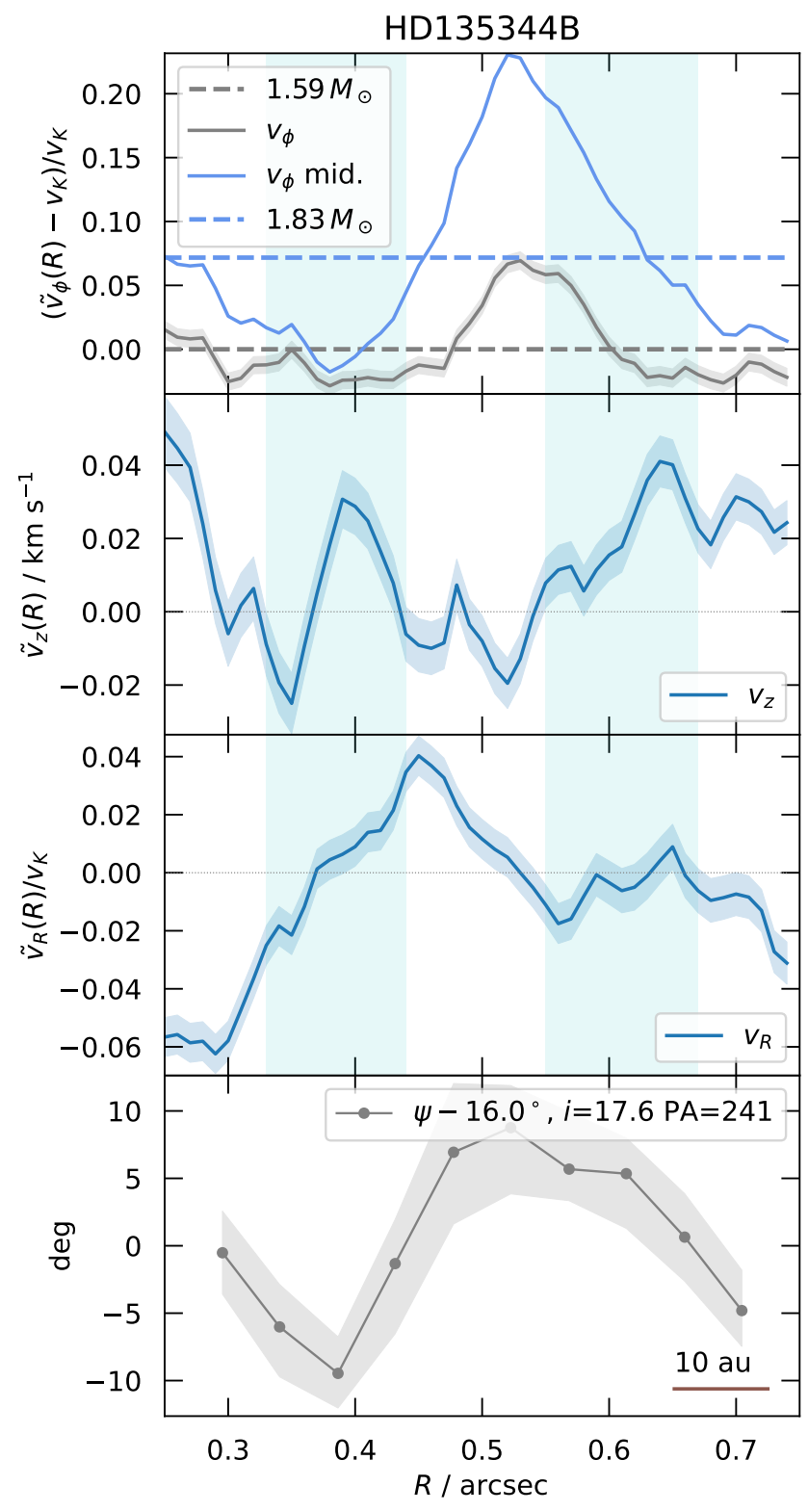

Figure 10. Rotation curve in HD 135344B. The regions in cyan correspond to the total extent of the two rings. From top to bottom, we show: 1) The azimuthal rotation curve $\tilde{v}_{\phi}(R)$. The dashed horizontal lines are comparison Keplerian profiles with the corresponding stellar mass. The curve labelled 'mid' is an extrapolation to the disc mid-plane assuming vertical Keplerian shear. 2): The vertical velocity component curve $\tilde{v}_{z}(R) .3$ ): The radial velocity component $\left.\tilde{v}_{r}(R) .4\right)$ : The opening angle of the cone tracing the unit opacity surface for ${ }^{12} \mathrm{CO}(2-1)$.

dispersion. The lack of a conspicuous spiral pattern in the rarer isotopologue maps, which are optically thinner, suggests that the spirals probably do not correspond to enhanced surface densities. These data tentatively support the case of "turbulent spirals", i.e. that turbulence, or superposed velocity components along the lineof-sight, is strong in this disc and particularly so in the spirals.

The uniform-slab diagnostics obtained with SLAB.LinE show that the grand-design 2-armed spiral in velocity-integrated intensity is neither seen in the gas surface density $\Sigma_{g}(\vec{x})$ nor in the gas temperature $T_{b}(\vec{x})$. Instead, it is reflected in the turbulent velocity map $v_{\text {turb }}(\vec{x})$. This is surprising as most attempts to constrain the level of turbulence in protoplanetary discs have resulted in upper limits, except in DM Tau (see Flaherty et al. 2020, and references therein), where values for $v_{\text {turb }}$ are similar as reported here for HD 135344B.

In the $\alpha$-viscosity model (Shakura \& Sunyaev 1973) viscosity is related to the thermal disc structure by a dimensionless parameter, $\alpha_{t}$. We can re-formulate our results by calculating this parameter locally, by comparing the turbulent velocities with the local sound speed, $\alpha_{t} \approx\left(v_{\text {turb }} / c_{s}\right)^{2}$. The uniform-slab diagnostic yields the gas temperature $T_{b}(\vec{x})$, from which we obtain the sound speed $c_{s}=$ $\sqrt{\gamma k_{B} T_{b} /\left(\mu m_{p}\right)}$, with an adiabatic index $\gamma=1.4$ and a molecular weight $\mu=2.3$. Fig. 9 shows that $\alpha_{t}(R)$ reaches values ${ }^{4}$ of $\sim 0.1$.

The inferred values for $\alpha_{t}$ seem very high, especially in the vortex interpretation of the large crescent modulating the outer ring. This is because very low levels of 'alpha' viscosity, of order $\alpha \sim 10^{-4}-10^{-3}$, are necessary to develop the Rossby-wave instability and explain the outer ring crescent with an anticyclonic vortex (e.g. Barge \& Sommeria 1995; Lyra \& Lin 2013; Zhu \& Stone 2014; Zhu \& Baruteau 2016). The turbulent velocity map in HD 135344B is thus unlikely to actually trace the source of viscosity in the disc, but may instead reflect the superposition of several velocity components, or superposed laminar flows along the line of sight.

Interestingly, the rotation curve of HD 135344B points at strong accretion towards the star, especially inside the inner dust ring (so $<0$.' 4). The radial velocity component, with a peak in absolute value $v_{R}=-0.37 \pm 0.04 \mathrm{~km} \mathrm{~s}^{-1}$ (Fig. 10), comes close to the sound speed, which ranges from $c_{S} \sim 0.55 \pm 0.04 \mathrm{~km} \mathrm{~s}^{-1}$ to $\sim 0.7 \pm 0.05 \mathrm{~km} \mathrm{~s}^{-1}$ over the radial domain plotted in Fig. 9. Transonic velocities are theoretically expected in the cavity of transition discs where magnetised winds result from thermal (photoevaporative) winds threaded by magnetic field lines, which exert a torque on the gas remaining in the wind and drive accretion (Wang \& Goodman 2017). The likelihood of photoevaporative winds and net poloidal fields in discs is theoretically established (e.g. Ercolano \& Pascucci 2017, and references therein).

We can estimate the corresponding accretion rate with $\dot{M}(R)=$ $-2 \pi R \Sigma_{g} \tilde{v}_{R}$. As illustrated in Fig. 9f, the peak accretion rate is $\dot{M}=$ $(2 \pm 0.1) \times 10^{-6} M_{\odot} \mathrm{yr}^{-1}$. This rate is 40 times larger than the stellar accretion rate of $5 \times 10^{-8} M_{\odot}{ }^{-1}$ (Fairlamb et al. 2015), and would deplete the disc mass sampled by $\mathrm{CO}(2-1)$, or $\sim 1.410^{-3} M_{\odot}$, in $\sim 700 \mathrm{yr}$ (the fraction of the material accreted by a potential planet inside the ring is small). It may be that the mass reservoir is not sampled by $\mathrm{CO}$, but even a very massive disc, with $0.1 M_{\odot}$, would still be much too short lived. The system may, perhaps, be observed in a transient phase, or an important fraction of the material being accreted inside $\sim 50$ au will eventually be expelled in a wind.

A similar issue as the disc depletion timescale arises with the diverging accretion rates near $50 \mathrm{au}$, so right on the inner dust ring (see Fig. 9f). Since the accretion rate is negative right outside the ring, and reaches $\sim(-1 \pm 0.2) 10^{-6} M_{\odot} \mathrm{yr}^{-1}$ at $\sim 60$ au, a gap would quickly develop at the location of the ring.

Another solution to both the very high values for $\alpha_{t}$, and the very high apparent accretion rate, is that accretion is restricted only to the surface layer, where the ${ }^{12} \mathrm{CO}(2-1)$ line originates. This would be a similar situation as that of meridional flows at the edges of a protoplanetary gap, but here in the case of the outer edge of the central cavity. The mid-plane, enclosing most of the disc mass, may instead be almost in pure azimuthal rotation, with at least a factor of 10

\footnotetext{
4 the steep rise towards the origin corresponds to the spurious inner ring in $v_{\text {turb }}$ that is due to confusion, as discussed in Sec. 3.3
} 

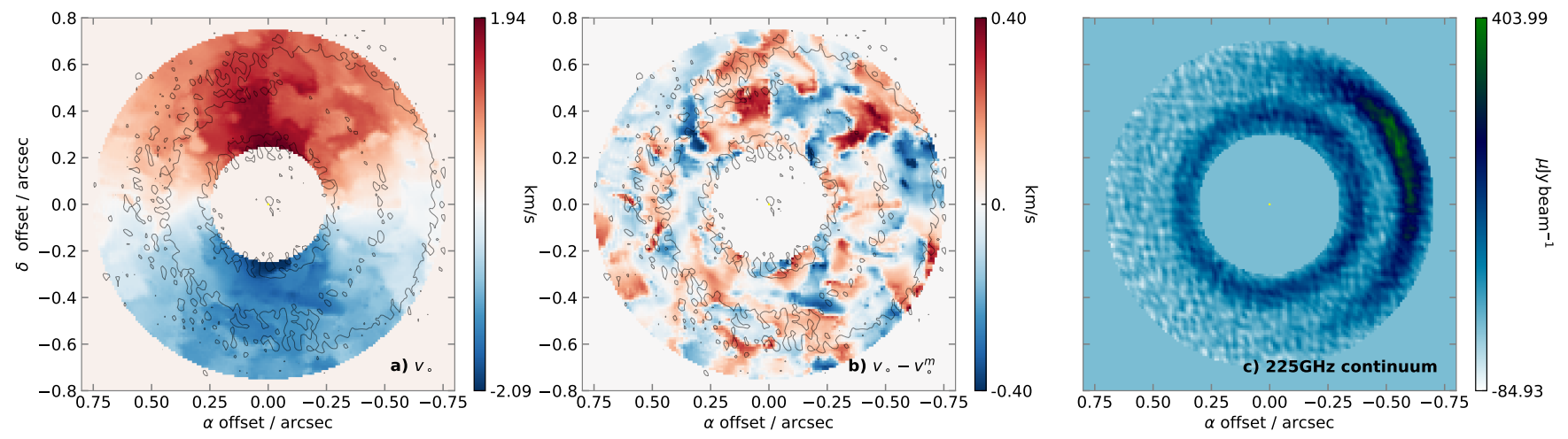

Figure 11. Face-on view of the kinematics in HD 135344B, inferred using ConeRot and the top-layer velocity centroid for the ${ }^{12} \mathrm{CO}(2-1) u v$-tapered datacube. a) Deprojected velocity centroid (approximating the disc with a cone). The contours trace the continuum image shown in c). b) Difference between the observed velocity field and the axially symmetric model, showing essentially thermal residuals. Deeper data are required to ascertain the structures seen near the position of the filament. c) Comparison with a face-on view of the continuum, deprojected as a thin disc with inclination $i=17.6 \mathrm{deg}$.

smaller radial velocity than in the surface so as to reconcile the disc accretion rate with the stellar accretion. If this is the case, then the origin of the line broadening could be the superposition of laminar flows along the line of sight, rather than genuine turbulence. The possibility of a strongly decreasing gradient for the radial velocity component towards the mid-plane can be tested with an extension of the rotation curve analysis from Sec. 3.4 to deeper CO isotopologue data. Theoretical models indeed suggest that both in the case of MRIdriven or wind-driven accretion, the active (accreting) layer in discs is expected to be confined to the surface (e.g. Mohanty et al. 2013; Bai 2016).

\section{CONCLUSIONS}

The HD 135344B disc is especially interesting in the context of planet-disc interactions. Here we reported on new ALMA observations, with unprecedented angular resolutions in this source. The data correspond to a partial delivery with about $1 / 10$ of the total integration, but nonetheless reveal interesting aspects of this disc, whose statistical significance will be further assessed pending delivery of the full dataset:

- A thin filament in the continuum image at $225 \mathrm{GHz}$ crosses the gap between the outer and inner rings (Fig. 1). Although its median intensity is at 4 times the noise level, confirmation of this filament requires a second epoch and deeper observations. The filament is found at the same location as the putative protoplanet proposed to be driving one of the spirals by Muto et al. (2012), and almost coaligned with a local twist in the IR spiral arm (same PA but radially shifted; Fig. 6c). We suggest that the radial shift is due to the curling of the spiral towards the star at the disc surface (Zhu et al. 2015).

- The ${ }^{12} \mathrm{CO}(2-1)$ velocity-integrated and dispersion maps trace the same spiral seen in scattered light, and characteristic of HD 135344B. The CO spiral is modulating an extended disc, and its arm-inter-am contrast is much shallower than in the near-IR. It is not affected by shadowing from a possible tilted inner disc. The spiral pattern and extended disc are absent from the peak intensity map, suggesting that the origin of the line broadening is turbulence rather than a temperature wave.

- The physical conditions and line-of-sight turbulent broadening $v_{\text {turb }}$ inferred from the uniform-slab and LTE approximations confirm that the extended disc is axially symmetric in the surface density of the CO layer, while $v_{\text {turb }}$ follows the IR spiral.

- The magnitude of $v_{\text {turb }}$ in this disc is very large, and close to $\sim 30 \%$ sonic. The correspond viscosity is $\alpha_{t} \sim 0.05-0.1$, which is much higher than standard values and suggests that the line broadening stems from superposed laminar flows rather than genuine turbulence.

- The disc rotation curve points at an inclination of $\sim 17 \mathrm{deg}$, which is consistent with estimates of the central star mass from the photospheric spectrum. If this inclination is correct, the inner ring in continuum emission is quite eccentric, with $e=0.14 \pm 0.04$, as the inclination required to circularise it is $i=23.8_{-2.6}^{2.5} \mathrm{deg}$.

- If the gaseous disc is axially symmetric, then its 3-D rotation curve, including radial and vertical axially symmetric flows, points at strong accretion inside the inner dust ring, within $\sim 0$.' 4 , with a radial velocity of up to $v_{R}=-0.2 \pm 0.02 \mathrm{~km} \mathrm{~s}^{-1}$. The corresponding mass accretion rate is $\dot{M}=(2 \pm 0.2) \times 10^{-6} M_{\odot} \mathrm{yr}^{-1}$, which may be reconciled with the $\sim 40$ times lower stellar accretion rate if only the surface layers are undergoing accretion.

\section{ACKNOWLEDGEMENTS}

S.C., S.P. and L.A.C., acknowledge support from Agencia Nacional de Investigación y Desarrollo de Chile (ANID) given by FONDECYT Regular grants 1171624, 1191934 and 1211656. N.M. acknowledges support from the Banting Postdoctoral Fellowships program, administered by the Government of Canada. B.E. acknowledges the support by the DFG Cluster of Excellence "Origin and Structure of the Universe" and of the DFG Research Unit "Transition Disks" grants FOR 2634/1, ER 685/8-1, and ER 685/9-1. VF's postdoctoral fellowship is supported by the Exoplanet Science Initiative at the Jet Propulsion Laboratory, California Institute of Technology, under a contract with the National Aeronautics and Space Administration (80NM0018D0004). MR acknowledges support from the FWO research program under project $1280121 \mathrm{~N}$.

\section{DATA AVAILABILITY}

The full ALMA dataset for project 2018.1.01066. S will be available on the ALMA archive, at the term of the proprietary period, one 
year after the project is either completed or terminated. Meanwhile, the reduced ALMA data underlying this article is available upon reasonable request to the corresponding author. The original analysis packages that sustain this work are publicly available at the following URLs:

https://github.com/simoncasassus/GMoments

https://github.com/simoncasassus/MPolarMaps

https://github.com/simoncasassus/ConeRot

https://github.com/simoncasassus/Slab

\section{AUTHOR CONTRIBUTIONS}

S.C: imaging, analysis, write-up, telescope proposal, software development: GMoments, MPolarMaps, ConeRot and Slab.Line. V.C: telescope proposal, spiral fits and comparisons. M.C.: uvmem imaging and software development. S.P.: IRDAP reduction, telescope proposal. P.W., C.B., D.P: planet-disc hydrodynamical context. B.E.: disc-wind context. N.vdM.: literature on HD 135344B, comparison with DALI model. R.D.: Estimate of ring eccentricity. O.A., M.R.: parallel direct imaging projects. All authors commented on the manuscript.

\section{REFERENCES}

ALMA Partnership et al., 2015, ApJ, 808, L3

Andrews S. M., et al., 2018, ApJ, 869, L41

Avenhaus H., Quanz S. P., Meyer M. R., Brittain S. D., Carr J. S., Najita J. R., 2014, ApJ, 790, 56

Avenhaus H., et al., 2018, ApJ, 863, 44

Bai X.-N., 2016, ApJ, 821, 80

Barge P., Sommeria J., 1995, A\&A, 295, L1

Baruteau C., Zhu Z., 2016, MNRAS, 458, 3927

Baruteau C., Wafflard-Fernandez G., Le Gal R., Debras F., Carmona A., Fuente A., Rivière-Marichalar P., 2021, arXiv e-prints, p. arXiv:2104.03769

Birnstiel T., Dullemond C. P., Pinilla P., 2013, A\&A, 550, L8

Boehler Y., Weaver E., Isella A., Ricci L., Grady C., Carpenter J., Perez L., 2017, ApJ, 840, 60

Boehler Y., et al., 2018, ApJ, 853, 162

Cárcamo M., Román P. E., Casassus S., Moral V., Rannou F. R., 2018, Astronomy and Computing, 22, 16

Carmona A., et al., 2014, A\&A, 567, A51

Carrasco-González C., et al., 2019, ApJ, 883, 71

Casassus S., Pérez S., 2019, ApJ, 883, L41

Casassus S., Stahl O., Wilson T. L., 2005, A\&A, 441, 181

Casassus S., Cabrera G. F., Förster F., Pearson T. J., Readhead A. C. S., Dickinson C., 2006, ApJ, 639, 951

Casassus S., et al., 2013, Nature, 493, 191

Casassus S., et al., 2015, ApJ, 812, 126

Casassus S., et al., 2018, MNRAS, 477, 5104

Casassus S., et al., 2019, MNRAS, 483, 3278

Cazzoletti P., et al., 2018, A\&A, 619, A161

Christiaens V., Casassus S., Perez S., van der Plas G., Ménard F., 2014, ApJ, 785, L12

Cieza L. A., et al., 2021, MNRAS, 501, 2934

Dong R., Hall C., Rice K., Chiang E., 2015, ApJ, 812, L32

Dong R., et al., 2018a, ApJ, 860, 124

Dong R., Najita J. R., Brittain S., 2018b, ApJ, 862, 103

Dullemond C. P., et al., 2018, ApJ, 869, L46

Ercolano B., Pascucci I., 2017, Royal Society Open Science, 4, 170114

Fairlamb J. R., Oudmaijer R. D., Mendigutía I., Ilee J. D., van den Ancker M. E., 2015, MNRAS, 453, 976

Flaherty K., et al., 2020, ApJ, 895, 109
Foreman-Mackey D., Hogg D. W., Lang D., Goodman J., 2013, PASP, 125, 306

Francis L., van der Marel N., 2020, ApJ, 892, 111

Gaia Collaboration et al., 2018, A\&A, 616, A1

Garg H., et al., 2020, arXiv e-prints, p. arXiv:2010.15310

Garufi A., Quanz S. P., Schmid H. M., Avenhaus H., Buenzli E., Wolf S., 2014, A\&A, 568, A40

Haugbølle T., Weber P., Wielandt D. P., Benítez-Llambay P., Bizzarro M., Gressel O., Pessah M. E., 2019, AJ, 158, 55

Isella A., Pérez L. M., Carpenter J. M., Ricci L., Andrews S., Rosenfeld K., 2013, ApJ, 775, 30

Isella A., Benisty M., Teague R., Bae J., Keppler M., Facchini S., Pérez L., 2019, ApJ, 879, L25

Jennings J., Booth R. A., Tazzari M., Rosotti G. P., Clarke C. J., 2020, MNRAS, 495, 3209

Koller J., Li H., Lin D. N. C., 2003, ApJ, 596, L91

Li H., Colgate S. A., Wendroff B., Liska R., 2001, ApJ, 551, 874

Lovelace R. V. E., Li H., Colgate S. A., Nelson A. F., 1999, ApJ, 513, 805

Lyra W., Lin M.-K., 2013, ApJ, 775, 17

Marino S., Casassus S., Perez S., Lyra W., Roman P. E., Avenhaus H., Wright C. M., Maddison S. T., 2015, ApJ, 813, 76

Mittal T., Chiang E., 2015, ApJ, 798, L25

Mohanty S., Ercolano B., Turner N. J., 2013, ApJ, 764, 65

Muto T., et al., 2012, ApJ, 748, L22

Muto T., et al., 2015, PASJ, 67, 122

Ohashi S., et al., 2020, ApJ, 900, 81

Pérez L. M., Isella A., Carpenter J. M., Chandler C. J., 2014, ApJ, 783, L13

Pérez S., Casassus S., Baruteau C., Dong R., Hales A., Cieza L., 2019, AJ, 158,15

Pinte C., et al., 2018, A\&A, 609, A47

Rafikov R. R., 2002, ApJ, 569, 997

Regály Z., Juhász A., Sándor Z., Dullemond C. P., 2012, MNRAS, 419, 1701

Schöier F. L., van der Tak F. F. S., van Dishoeck E. F., Black J. H., 2005, A\&A, 432, 369

Shakura N. I., Sunyaev R. A., 1973, A\&A, 24, 337

Sierra A., Lizano S., Barge P., 2017, ApJ, 850, 115

Sierra A., Lizano S., Macías E., Carrasco-González C., Osorio M., Flock M., 2019, ApJ, 876, 7

Stahl O., Casassus S., Wilson T., 2008, A\&A, 477, 865

Stolker T., et al., 2016, A\&A, 595, A113

Stolker T., et al., 2017, ApJ, 849, 143

Teague R., et al., 2016, A\&A, 592, A49

Teague R., Bae J., Bergin E. A., 2019, Nature, 574, 378

Varnière P., Tagger M., 2006, A\&A, 446, L13

Wang L., Goodman J. J., 2017, ApJ, 835, 59

Weber P., Benítez-Llambay P., Gressel O., Krapp L., Pessah M. E., 2018, ApJ, 854, 153

Weidenschilling S. J., 1977, MNRAS, 180, 57

Wichittanakom C., Oudmaijer R. D., Fairlamb J. R., Mendigutía I., Vioque M., Ababakr K. M., 2020, MNRAS, 493, 234

Wilson T. L., Rood R., 1994, ARA\&A, 32, 191

Xie C., Ren B., Dong R., Pueyo L., Ruffio J.-B., Fang T., Mawet D., Stolker T., 2021, ApJ, 906, L9

Yen H.-W., Gu P.-G., 2020, ApJ, 905, 89

Zhu Z., Baruteau C., 2016, MNRAS, 458, 3918

Zhu Z., Stone J. M., 2014, ApJ, 795, 53

Zhu Z., Dong R., Stone J. M., Rafikov R. R., 2015, ApJ, 813, 88

de Val-Borro M., Artymowicz P., D’Angelo G., Peplinski A., 2007, A\&A, 471,1043

van Holstein R. G., et al., 2020, A\&A, 633, A64

van der Marel N., et al., 2013, Science, 340, 1199

van der Marel N., van Dishoeck E. F., Bruderer S., Pérez L., Isella A., 2015a, A\&A, 579, A106

van der Marel N., Pinilla P., Tobin J., van Kempen T., Andrews S., Ricci L., Birnstiel T., 2015b, ApJ, 810, L7

van der Marel N., van Dishoeck E. F., Bruderer S., Andrews S. M., Pontoppidan K. M., Herczeg G. J., van Kempen T., Miotello A., 2016a, A\&A, 585, A58 
van der Marel N., Cazzoletti P., Pinilla P., Garufi A., 2016b, ApJ, 832, 178

van der Marel N., et al., 2021, AJ, 161, 33

van der Plas G., Ménard F., Canovas H., Avenhaus H., Casassus S., Pinte C.,

Caceres C., Cieza L., 2017, A\&A, 607, A55

\section{APPENDIX A: CHANNEL MAPS}

Channels maps for the tclean image reconstructions of the ${ }^{12} \mathrm{CO}(2-$ 1) data are shown in Fig. A1.

\section{APPENDIX B: DISC ORIENTATION FROM THE CONTINUUM}

disc orientation is often inferred from continuum data under the assumption of axial symmetry, either directly from visibility data (e.g. Jennings et al. 2020), or simply by fitting projected elliptical Gaussians in the image plane. Here we follow an image plane approach, under the assumption of axial symmetry for a thin disc (with null aspect ratio). We minimise the variance in the radial profile for the continuum intensity $I_{v}(R, \phi)$,

$\chi_{\text {var }}^{2}=\frac{1}{I_{\text {noise }}^{2}} \sum_{l=l_{1}}^{l=l_{2}} \sigma_{\phi}^{2}\left(R_{l} ; \mathrm{PA}, i, \Delta \alpha, \Delta \delta\right)$,

where $I_{\text {noise }}$ is the thermal noise in the image, and where $\sigma_{\phi}^{2}$ is the azimuthal variance of intensities,

$\sigma_{\phi}^{2}\left(R_{l}\right)=\frac{1}{N_{\phi}} \sum_{k=0}^{N_{\phi}-1}\left(I_{v}\left(R_{l}, \phi_{k}\right)-\left\langle I_{v}\left(R_{l}\right)\right\rangle\right)^{2}$,

with

$\left\langle I_{v}\left(R_{l}\right)\right\rangle=\frac{1}{N_{\phi}} \sum_{k=0}^{N_{\phi}-1} I_{v}\left(R_{l}, \phi_{k}\right)$.

The radial profile $\left\langle I_{\nu}(R)\right\rangle$ and the variance $\sigma_{\phi}^{2}(R)$ profile depend on the disc position angle, inclination, and choice of origin for the (standard) polar expansion.

The minimisation of $\chi_{\mathrm{var}}^{2}(\mathrm{PA}, i, \Delta \alpha, \Delta \delta)$ in Eq. B1 is carried out with the EMCEE package (Foreman-Mackey et al. 2013), with flat priors, and with 600 iterations and 40 walkers. The resulting posterior distributions are summarised in Fig. B1 for an application to HD 135344B. This strategy to infer the disc orientation is implemented using Python in the MPolarMaps package, and is publicly available at git@github.com:simoncasassus/ MPolarMaps.git.

This paper has been typeset from a $\mathrm{T}_{\mathrm{E}} \mathrm{X} / \mathrm{L} \mathrm{T} \mathrm{E} \mathrm{X}$ file prepared by the author. 


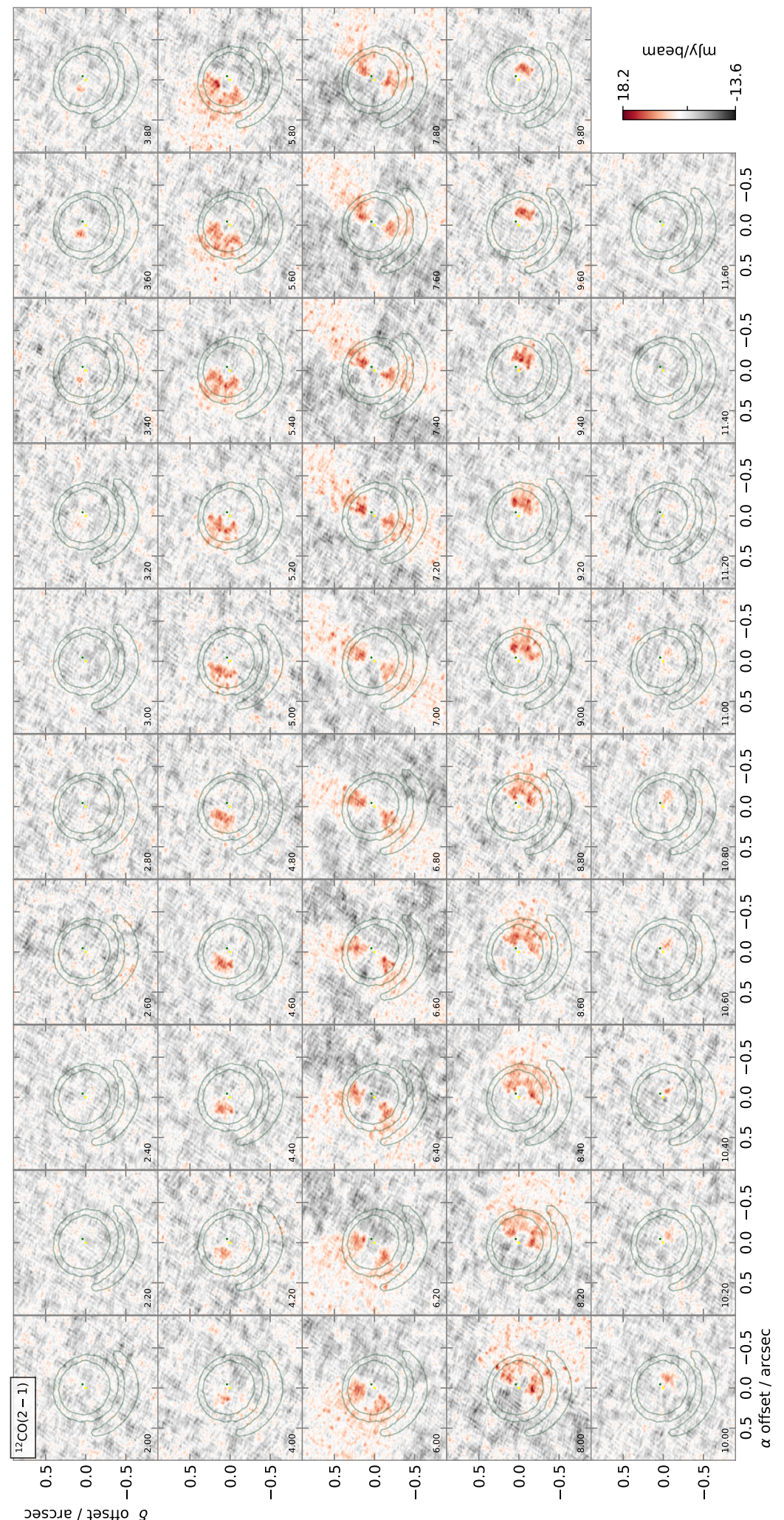

Figure A1. Channel maps from the tclean ${ }^{12} \mathrm{CO}(2-1)$ datacube. The continuum is outlined in contours. 


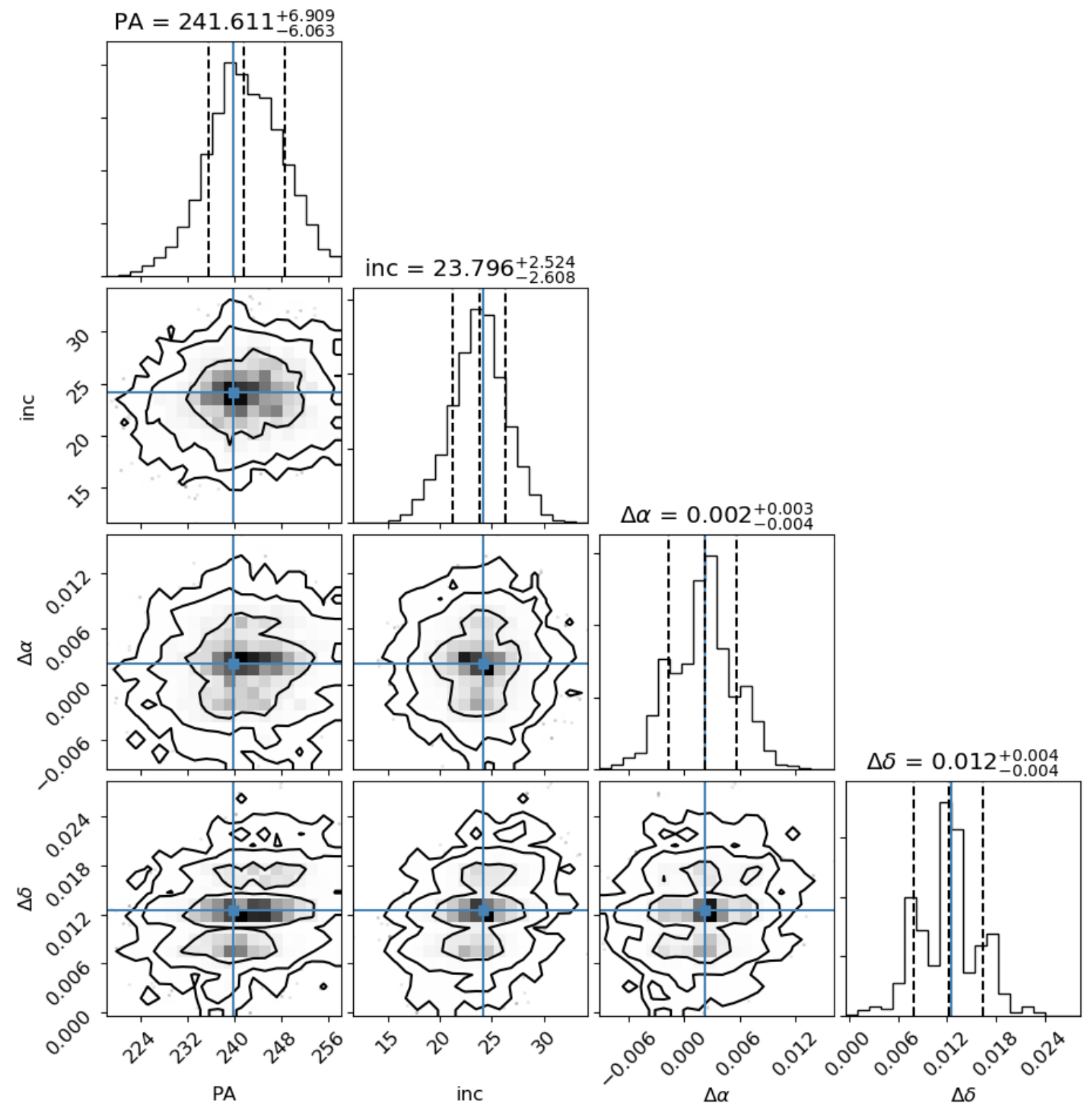

Figure B1. Corner plot from the $\chi_{\mathrm{var}}^{2}$ optimisation of the orientation parameters in HD 135344B using the tclean continuum image at $225 \mathrm{GHz}$ continuum from Fig. $1 \mathrm{~b}$ 